\title{
Free Energy and Magnetic Penetration Depth of a $d$-Wave Superconductor in the Meissner State
}

\author{
Mei-Rong Li, ${ }^{a, b}$ P.J. Hirschfeld ${ }^{c}$ and P. Wölfle $\mathrm{e}^{a}$ \\ ${ }^{a}$ Institut für Theorie der Kondensierten Materie, Universität Karlsruhe, 76128 Karlsruhe, Germany \\ ${ }^{b}$ Department of Physics, Nanjing University, Nanjing 210093, P.R.China \\ ${ }^{c}$ Department of Physics, University of Florida, Gainesville, FL 32611, USA
}

\begin{abstract}
We investigate the free energy and the penetration depth of a quasi-two-dimensional $d$-wave superconductor in the presence of a weak magnetic field by taking account of thermal, nonlocal, and nonlinear effects. In an approximation in which the superfluid velocity $v_{s}$ is assumed to be slowly varying, the free energy is calculated and compared with available results in several limiting cases. It is shown that either nonlocal or nonlinear effects may cut off the linear- $T$ dependence of both the free energy and the penetration depth in all the experimental geometries. At extremely low $T$, the nonlocal effects will also generically modify the linear $H$ dependence of the penetration depth ("nonlinear Meissner effect") in most experimental geometries, but for supercurrents oriented along the nodal directions, the effect may be recovered. We compare our predictions with existing experiments on the cuprate superconductors.
\end{abstract}

PACS Numbers: 74.25.Nf, 74.20.Fg

\section{INTRODUCTION}

Many unusual aspects of the unconventional superconductivity in the cuprate and heavy fermion materials are related to the existence of nodes in the energy gap. Although intensely discussed in the 1980s and 1990s, most of these phenomena were already known much earlier to students of the unconventional superfluid ${ }^{3} \mathrm{He}$, which exhibits several phases, some with gap nodes and accompanying low-energy fermionic excitations. For example, power laws in specific heat, transport properties, and NMR were predicted and measured in the anisotropic, high-pressure "A" phase, along with singular responses to impurities, unusual vortex phases, and even anomalous Josephson currents [1]. From this perspective, the one important difference between the new superconductors and the ${ }^{3} \mathrm{He}$ system is that they are charged; unconventional superconductivity can be uniquely probed by studying the response of the system to an applied electromagnetic field.

Even the simplest such phenomenon, the expulsion of a weak applied magnetic field, or Meissner effect, is still the subject of intense discussion today in the context of unconventional superconductivity. The new materials are strong type-II superconductors, with large values of the London penetration depth $\lambda_{0}$ and small coherence lengths $\xi_{0}$, such that the condition $\lambda_{0} \gg \xi_{0}$ is expected to hold. It was therefore anticipated in the earliest theories of the Meissner effect in unconventional superconductors [2] that the electrodynamics of the system could be treated as local, i.e., as though the Cooper pairs were point objects, neglecting the spatial variation of the electromagnetic wave over the extent of the pair. Under this rather plausible assumption, the simple anisotropic extension of the BCS theory is expected to hold,

$$
\frac{\lambda_{0}^{2}}{\lambda^{2}(T)}=1-\left\langle\hat{k}_{\|}^{2} \int d \xi_{k}\left(-\frac{\partial f}{\partial E_{k}}\right)\right\rangle_{\mathrm{FS}},
$$

where the quasiparticle spectrum is $E_{k}=\sqrt{\xi_{k}^{2}+\Delta_{k}^{2}}, \xi_{k}$ is the normal-state electron band, $\Delta_{k}$ is the momentumdependent order parameter, $f$ is the Fermi function, $\hat{k}_{\|}$is the direction of the supercurrent, and $\langle\cdots\rangle_{F S}$ represents a Fermi surface average. The London penetration depth at $T=0$ is given by $\lambda_{0} \equiv \sqrt{m c^{2} /\left(4 \pi n e^{2}\right)}$, and is typically one to several thousand Angström in the new materials.

At low temperatures, the local penetration depth calculated from Eq. (1) may be shown [2] to vary as a power law of temperature, $\lambda(T)-\lambda_{0} \sim\left(T / \Delta_{0}\right)^{\alpha}$, where $\Delta_{0}$ is the gap maximum and the exponent $\alpha$ depends on dimensionality, nodal topology, and the rate at which the gap goes to zero near a nodal wave vector $\mathbf{k}_{n}$. For line nodes in a threedimensional (3D) system (or point nodes in a 2D system) like the $d_{x^{2}-y^{2}}$ state thought to characterize the cuprate superconductors, $\alpha$ is found to be 1. Thus when Hardy et al. [3] measured a linear temperature dependence in the $\mathrm{YBa}_{2} \mathrm{Cu}_{3} \mathrm{O}_{6.95}$ (YBCO) system down to a few degrees $\mathrm{K}$, it was dramatic evidence in favor of line order parameter nodes and possible unconventional pairing. 
Recently, Kosztin and Leggett [4] questioned the theoretical basis for this identification, pointing out that the approximation of local electrodynamics used to derive Eq. (1) is not valid for the quasiparticle states near the nodes whose occupation determines the asymptotic low-temperature behavior. The coherence length $\xi_{0} \equiv v_{F} / \pi \Delta_{0}$ determines the scale of order-parameter variations, as in the usual BCS theory, but an effective coherence length $\xi_{0 k} \equiv v_{F} / \pi \Delta_{k}$ appears in the electromagnetic response, as noted in Ref. [5. The divergence of $\xi_{0 k}$ at the nodes means that quasiparticle states near the nodes must always be treated nonlocally [6]. A full nonlocal calculation gives a crossover from a $\delta \lambda \sim T$ regime for $E_{\text {nonloc }} \ll T \ll \Delta_{0}$ to a $\delta \lambda \sim T^{2}$ for $T \ll E_{\text {nonloc }}$. In the YBCO system, this crossover scale $E_{\text {nonloc }} \simeq \Delta_{0} \xi_{0} / \lambda_{0}$ is about $1 \mathrm{~K}$, and thus the identification of the $d$-wave state on the basis of the local theory is not really in question; measurements on the purest YBCO crystals down to $1 \mathrm{~K}$ or so have seen no deviation from the linear behavior. [7] Quadratic dependences $\delta \lambda \sim T^{2}$ measured in some samples are more likely due to impurity effects [2,8,9], which also produce a $T \rightarrow T^{2}$ crossover.

An interesting perspective on the question of the linear $T$ dependence of the penetration depth was provided recently by Schopohl and Dolgov [10], who observed that if the $d$-wave penetration depth temperature dependence were to remain linear down to $T=0$, one would be unable to satisfy the third law of thermodynamics (Nernst's theorem). In the framework of linear-response theory, they expressed the change of the free energy in the presence of the magnetic field (for fixed external current source) as a functional of the penetration depth,

$$
\Delta \mathcal{F}^{(S D)}=-\frac{1}{8 \pi} \int \frac{d^{3} q}{(2 \pi)^{3}}\left[q^{2}+\frac{1}{\lambda^{2}(\mathbf{q}, T)}\right]\left|\mathbf{A}_{\mathbf{q}}(T)\right|^{2},
$$

where $\mathbf{A}_{\mathbf{q}}(T)$ is the Fourier component of the magnetic vector potential. The resulting entropy is

$$
S^{(S D)}(T)=-\frac{1}{8 \pi} \int \frac{d^{3} q}{(2 \pi)^{3}} \frac{\partial}{\partial T}\left[\frac{1}{\lambda^{2}(\mathbf{q}, T)}\right]\left|\mathbf{A}_{\mathbf{q}}(T)\right|^{2} .
$$

The Nernst theorem requires $S$ to be 0 when $T \rightarrow 0$, inconsistent with $\lambda(T) \sim T$.

Schopohl and Dolgov speculated that this result might imply the instability of a pure $d$-wave state within BCS theory at sufficiently low temperatures. It was pointed out in Ref. [11], however, that the extra magnetic-field-induced quasiparticles that deplete the shielding current, leading to a magnetic-field-dependent penetration depth known as the nonlinear Meissner effect [12,13], were neglected in this analysis. Several authors have recently considered the effects of these nonlinear terms in the supercurrent on transverse magnetization measurements [14] and on the structure of the vortex lattice [15, 16]. The basic idea is that, like impurity effects, the magnetic field itself may serve as a Cooper pair breaker that creates nodal quasiparticles, leading to a temperature dependent penetration $\operatorname{depth} \delta \lambda(T) \simeq T^{2}$ at temperatures below the scale for nonlinear electrodynamics, $E_{\text {nonlin }} \simeq v_{s} k_{F}$ with $v_{s}$ a typical supercurrent velocity and $k_{F}$ the Fermi wave vector. The current authors [17] then provided a synthesis of the various arguments given above, pointing out that for any experimental geometry with given disorder, external current distribution, and GinzburgLandau parameter $\kappa$, a sample of $d$-wave superconductor would inevitably avoid the violation of the Nernst theorem by creating a $T^{2}$ term in its penetration depth through a competition of nonlinear, nonlocal, and impurity effects.

Although this issue of principle has been resolved, an important prediction of the $d$-wave model for the electrodynamics of high- $T_{c}$ superconductors (HTS) has not yet been confirmed. In the early discussion of the symmetry of the HTS order parameter, Yip and Sauls proposed that the angular position of the gap nodes could be probed by a measurement of the magnetic field dependence of the penetration depth. In the local limit and for $T \rightarrow 0$, they predicted that the nonlinear effect induces a linear- $H$ term in the penetration depth,

$$
\frac{\delta \lambda(H)}{\lambda(0)}=\frac{\lambda(H)-\lambda(0)}{\lambda(0)} \simeq \begin{cases}H / H_{0} & \mathbf{v}_{s} \| \text { node } \\ \frac{1}{\sqrt{2}} H / H_{0} & \mathbf{v}_{s} \| \text { antinode }\end{cases}
$$

where $H$ is the applied magnetic field at the surface and

$$
H_{0}=\frac{3 \Phi_{0}}{\pi^{2} \xi_{0} \lambda_{0}}
$$

is of order the thermodynamic critical field of the system with $\Phi_{0}$ the flux quantum. The prefactor of the linear $H$ term in Eq. (4) depends generally on the angle $\theta$ that the supercurrent makes with the crystalline $\hat{a}$ axis and is independent of $T$ (the $T$ dependence appears in the $H^{2}$ term as discussed recently by Dahm and Scalapino [38]). Several experimental groups tried to verify the prediction of Yip and Sauls, but failed to identify a linear $H$ term which scaled with the temperature according to the theory [18 20]. 
There are three relevant energy scales in the low-energy sector in the Meissner state, $T, E_{\text {nonlin }}$, and $E_{\text {nonloc }}$. The first two are experimentally controlled parameters while the last is an intrinsic one. Obviously, $\lambda(T) \sim T$ behavior should be expected at $T \gg E_{\text {nonloc }}, E_{\text {nonlin. }}$. But in the opposite limit, i.e., at extremely low $T$, either nonlinear or nonlocal effects may play a crucial role in modifying the linear $T$ behavior. Thus a full study including both nonlinear and nonlocal effects in the Meissner state is necessary. A similar investigation has been performed by Amin, Affleck, and Franz [15] in the mixed state where $H \geq H_{c 1}$, the lower critical field. It was found in this numerical study that the nonlinear and nonlocal corrections were of the same order. This result is not surprising: in the mixed state the nonlinear energy scale and the nonlocal one are not independent, since typical spatial variations take place on a scale of the magnetic length or intervortex distance, fixed by the fluxoid quantization.

In this paper, we perform a full study of a quasi-two-dimensional (2D) $d$-wave superconductor in the presence of a weak magnetic field. We start from a phenomenological BCS Hamiltonian with a quasiparticle energy spectrum in the normal state, $\xi_{\mathbf{k}}=\epsilon_{\mathbf{k}}-\mu$ ( $\mu$ is the chemical potential) and a $d_{x^{2}-y^{2}}$-wave order parameter, $\Delta_{\varphi}=\Delta_{0} \cos 2 \varphi(\varphi$ is the angle of $\mathbf{k}$ with the $\hat{a}$ axis). Using the functional-integral approach, we calculate the free energy and compare it with that obtained in other theories. Our main approximation is that the supercurrent along the surface boundary is a slowly varying function of the distance to the surface. Thus we take $\mathbf{v}_{s}=(e / m c) \mathbf{A}_{\mathbf{q}=0}$ as the supercurrent to which the quasiparticles couple, leading to the Doppler shift in the energy spectrum of particles and holes $\pm E_{\mathbf{k}}+\mathbf{k} \cdot \mathbf{v}_{s}$, where $E_{\mathbf{k}}=\left[\xi_{k}^{2}+\Delta_{\varphi}^{2}\right]^{1 / 2}$. The response of the quasiparticles to the $\mathbf{A}_{\mathbf{q} \neq 0}$ modes is treated perturbatively, and the resulting response function involves both the nonlinear and nonlocal effects. From this response function we then calculate the penetration depth, and express it as a scaling function of the two parameters $E_{\text {nonloc }} / T, E_{\text {nonlin }} / T$. We show explicitly that, below a temperature $T^{*}$, the linear $T$ behavior of the penetration depth is always modified by either nonlinear or nonlocal effects to a quadratic function of $T$, while the linear $H$ behavior in the penetration depth is also cut off by the nonlocal effects when $H$ is below some characteristic critical field $H^{*}$, which is geometry dependent. For most geometries $H^{*} \simeq H_{c 1}$, implying the unobservability of the nonlinear Meissner effect, as discussed in our recent paper on this subject [22]. But for some special geometries, in which the supercurrent is along nodal directions, the nonlinear Meissner effect may be recovered. We also present a brief discussion on the geometry in which the magnetic field is along the conduction plane, which presents some special features due to the highly layered nature of the cuprates. Finally, we investigate the consequence of the spatially varying nature of the supercurrent and find that this property does not influence the qualitative behavior of the penetration depth.

The paper is organized as follows. In Sec. II, the model Hamiltonian and the partition function are obtained. In Sec. III, the expression of the free energy as well as its comparison with various theories in several limiting cases is presented, and the supercurrent and the response function are discussed. Detailed study of the penetration depth in the presence of a constant $v_{s}$ is given in Sec. IV. Section V is devoted to concluding remarks and comparison with existing experiments. Finally, a discussion of the renormalization of the penetration depth due to space-dependent $v_{s}$ is included in the Appendix.

\section{MODEL HAMILTONIAN AND PARTITION FUNCTION}

We start from a phenomenological $d_{x^{2}-y^{2}}$-wave BCS mean-field Hamiltonian of a 2D system in the presence of a weak magnetic field [23]:

$$
\begin{aligned}
\widehat{H}_{\mathrm{MF}}= & \sum_{\sigma} \int d^{2} \mathbf{r} c_{\sigma}^{\dagger}(\mathbf{r})\left\{\frac{1}{2 m}\left[-i \vec{\nabla}-\frac{e}{c} \mathbf{A}(\mathbf{r})\right]^{2}-\mu\right\} c_{\sigma}(\mathbf{r})+\int d^{2} \mathbf{r} d^{2} \mathbf{r}^{\prime}\left[\Delta\left(\mathbf{r}, \mathbf{r}^{\prime}\right) c_{\uparrow}^{\dagger}(\mathbf{r}) c_{\downarrow}^{\dagger}\left(\mathbf{r}^{\prime}\right)+\text { H.c. }\right] \\
& +\int d^{2} \mathbf{r} d^{2} \mathbf{r}^{\prime} V\left(\mathbf{r}-\mathbf{r}^{\prime}\right)\left|b\left(\mathbf{r}, \mathbf{r}^{\prime}\right)\right|^{2},
\end{aligned}
$$

where $-V\left(\mathbf{r}-\mathbf{r}^{\prime}\right)<0$ is the effective attraction between electrons responsible for the $d_{x^{2}-y^{2}}$ pairing, $\Delta\left(\mathbf{r}, \mathbf{r}^{\prime}\right)=$

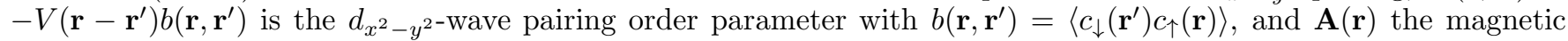
vector potential. It is well known that in the Meissner state the supercurrent formed by the Cooper pairs effectively screens the magnetic field from penetrating into the bulk of the sample. Within the penetration depth away from the surface, both magnetic field and the supercurrent decay with the distance to the surface, and the pairing order parameter acquires, in principle, a spatially varying phase. As usual we write $\Delta\left(\mathbf{r}, \mathbf{r}^{\prime}\right)$ as $\widetilde{\Delta}\left(\mathbf{r}-\mathbf{r}^{\prime}\right) e^{i \phi(\mathbf{R})}$ with a real $\widetilde{\Delta}\left(\mathbf{r}-\mathbf{r}^{\prime}\right)$ and $\mathbf{R}=\left(\mathbf{r}+\mathbf{r}^{\prime}\right) / 2$. Note we neglect the supression of the magnitude $\widetilde{\Delta}$ at surface of the sample, since we expect these corrections to the penetration depth will be small in the limit $\lambda_{0} \gg \xi_{0}$ of interest.

The partition function of Hamiltonian (6) is 


$$
\mathcal{Z}=\mathcal{Z}_{0} \int[D d]\left[D d^{*}\right] \exp \left\{-\int_{0}^{1 / T} d \tau\left[\sum_{\alpha, \beta=1}^{2} \int d^{2} \mathbf{r} d^{2} \mathbf{r}^{\prime} d_{\alpha}^{*}(\mathbf{r}) \mathcal{M}_{\alpha \beta}\left(\mathbf{r}, \mathbf{r}^{\prime}\right) d_{\beta}\left(\mathbf{r}^{\prime}\right)\right]\right\}
$$

where

$$
\mathcal{Z}_{0}=\exp \left[-\frac{1}{T}\left(\frac{N_{s} e^{2}}{2 m c^{2}} \int d^{2} \mathbf{r} A^{2}(\mathbf{r})+\frac{N_{s} \Delta_{0}^{2}}{V_{0}}\right)\right]
$$

with $N_{s}$ the total number of superconducting electrons, $d_{1}(\mathbf{r})=c_{\uparrow}(\mathbf{r}), d_{2}(\mathbf{r})=c_{\downarrow}^{*}(\mathbf{r})$, and $\mathcal{M}_{\alpha \beta}\left(\mathbf{r}, \mathbf{r}^{\prime}\right)$ is a matrix element of the $2 \times 2$ matrix $\widehat{\mathcal{M}}$ in the Nambu representation,

$$
\widehat{\mathcal{M}}\left(\mathbf{r}, \mathbf{r}^{\prime}\right)=\left(\begin{array}{cc}
\left\{\partial_{\tau}+\frac{1}{2 m}\left[i \vec{\nabla}+\frac{e}{c} \mathbf{A}(\mathbf{r})\right]^{2}-\mu\right\} \delta\left(\mathbf{r}-\mathbf{r}^{\prime}\right) & \widetilde{\Delta}\left(\mathbf{r}-\mathbf{r}^{\prime}\right) e^{i \phi(\mathbf{R})} \\
\widetilde{\Delta}\left(\mathbf{r}-\mathbf{r}^{\prime}\right) e^{-i \phi(\mathbf{R})} & \left\{\partial_{\tau}-\frac{1}{2 m}\left[i \overleftarrow{\nabla}+\frac{e}{c} \mathbf{A}(\mathbf{r})\right]^{2}+\mu\right\} \delta\left(\mathbf{r}^{\prime}-\mathbf{r}\right)
\end{array}\right)
$$

The use of a unitary transformation

$$
\widehat{U}\left(\mathbf{r}, \mathbf{r}^{\prime}\right)=\left(\begin{array}{cc}
e^{i \phi(\mathbf{R}) / 2} & 0 \\
0 & e^{-i \phi(\mathbf{R}) / 2}
\end{array}\right)
$$

to $\widehat{\mathcal{M}}$ is made for eliminating the phase factor of $\Delta\left(\mathbf{r}, \mathbf{r}^{\prime}\right)$, leading to

$$
\begin{aligned}
& \widehat{\widetilde{\mathcal{M}}}\left(\mathbf{r}, \mathbf{r}^{\prime}\right)=\widehat{U}^{\dagger}\left(\mathbf{r}, \mathbf{r}^{\prime}\right) \widehat{\mathcal{M}}\left(\mathbf{r}, \mathbf{r}^{\prime}\right) \widehat{U}\left(\mathbf{r}, \mathbf{r}^{\prime}\right) \\
& =\left(\begin{array}{cc}
\left\{\partial_{\tau}+\left[\frac{(i \vec{\nabla})^{2}}{2 m}-\mu\right]+\mathbf{v}_{s}(\mathbf{r}) \cdot i \vec{\nabla}+\frac{1}{2} m v_{s}^{2}(\mathbf{r})\right\} \delta\left(\mathbf{r}-\mathbf{r}^{\prime}\right) & \widetilde{\Delta}\left(\mathbf{r}-\mathbf{r}^{\prime}\right) \\
\widetilde{\Delta}\left(\mathbf{r}-\mathbf{r}^{\prime}\right) & \left\{\partial_{\tau}-\left[\frac{(i \overleftarrow{\nabla})^{2}}{2 m}-\mu\right]-i \overleftarrow{\nabla} \cdot \mathbf{v}_{s}(\mathbf{r})-\frac{1}{2} m v_{s}^{2}(\mathbf{r})\right\} \delta\left(\mathbf{r}^{\prime}-\mathbf{r}\right)
\end{array}\right),
\end{aligned}
$$

where

$$
\mathbf{v}_{s}(\mathbf{r})=\frac{e \mathbf{A}(\mathbf{r})}{m c}-\frac{\nabla \phi(\mathbf{r})}{2 m}
$$

is the supercurrent velocity. In writing down Eq. (11) we have used the relation $\vec{\nabla} \cdot \mathbf{v}_{s}(\mathbf{r})=0$ which corresponds to the conservation of the supercurrent. $\mathbf{v}_{s}(\mathbf{r})$ should be gauge invariant, which is guaranteed by the compensation of the two terms in the right-hand side of Eq. (12) under a gauge transformation. It is necessary to fix the gauge before proceeding. In the Meissner state the most convenient gauge choice is the London gauge in which $\nabla \phi(\mathbf{r})=0$, and hence

$$
\mathbf{v}_{s}(\mathbf{r})=\frac{e \mathbf{A}(\mathbf{r})}{m c}, \quad \nabla \cdot \mathbf{A}(\mathbf{r})=0 .
$$

The partition function $\mathcal{Z}$ in Eq. (7) can be expressed in momentum space as

$$
\mathcal{Z}=\mathcal{Z}_{0} \exp \left[\sum_{n} \operatorname{tr} \ln \left(\widehat{Q}^{(1)}\left(i \omega_{n}\right)+\widehat{Q}^{(2)}\right)\right]
$$

where $\omega_{n}$ is the fermion Matsubara frequency,

$$
\begin{gathered}
\widehat{Q}_{\mathbf{k}, \mathbf{k}^{\prime}}^{(1)}\left(i \omega_{n}\right)=\left(\begin{array}{cc}
-i \omega_{n}+\xi_{\mathbf{k}}+M_{\mathbf{k}, \mathbf{k}} & \Delta_{\mathbf{k}} \\
\Delta_{\mathbf{k}} & -i \omega_{n}-\xi_{\mathbf{k}}-M_{-\mathbf{k},-\mathbf{k}}
\end{array}\right) \delta_{\mathbf{k k}^{\prime}}=-\widehat{\mathcal{G}}^{-1}\left(i \omega_{n}, \mathbf{k}\right) \delta_{\mathbf{k k}^{\prime}}, \\
\widehat{Q}_{\mathbf{k}, \mathbf{k}^{\prime}}^{(2)}=\left(\begin{array}{cc}
M_{\mathbf{k}, \mathbf{k}^{\prime}} & 0 \\
0 & -M_{-\mathbf{k}^{\prime},-\mathbf{k}}
\end{array}\right) .
\end{gathered}
$$

with $\Delta_{\mathbf{k}}=\int d^{2} \mathbf{r} \tilde{\Delta}(\mathbf{r}) \exp (i \mathbf{k} \cdot \mathbf{r}) \simeq \Delta_{0} \cos 2 \varphi$ on the Fermi surface, $M_{\mathbf{k}_{1}, \mathbf{k}_{2}}=-(e / m c) \mathbf{k}_{2} \cdot \mathbf{A}_{\mathbf{k}_{1}-\mathbf{k}_{2}}+\left(e^{2} / 2 m c^{2}\right) \sum_{\mathbf{p}} \mathbf{A}_{\mathbf{p}}$. $\mathbf{A}_{\mathbf{k}_{1}-\mathbf{k}_{2}-\mathbf{p}}$ with $\mathbf{A}_{\mathbf{k}}$ the Fourier component of $\delta \mathbf{A}(\mathbf{r})=\mathbf{A}(\mathbf{r})-\overline{\mathbf{A}}\left(\overline{\mathbf{A}}\right.$ is the spatial average), $\xi_{\mathbf{k}}=\epsilon_{\mathbf{k}}-\mu$ is the energy spectrum of an electron in the normal state, and $\widehat{\mathcal{G}}\left(i \omega_{n}, \mathbf{k}\right)$ is Green's function matrix in Nambu's representation 


$$
\widehat{\mathcal{G}}\left(i \omega_{n}, \mathbf{k}\right)=-\left(\begin{array}{cc}
\frac{i \omega_{n}+\xi_{\mathbf{k}}+M_{-\mathbf{k},-\mathbf{k}}}{W_{\mathbf{k}}} & \frac{\Delta_{\mathbf{k}}}{W_{\mathbf{k}}} \\
\frac{\Delta_{\mathbf{k}}}{W_{\mathbf{k}}} & \frac{i \omega_{n}-\xi_{\mathbf{k}}-M_{\mathbf{k}, \mathbf{k}}}{W_{\mathbf{k}}}
\end{array}\right)
$$

Here

$$
W_{\mathbf{k}}=-\left(i \omega_{n}+\mathbf{k} \cdot \mathbf{v}_{s}\right)^{2}+E_{\mathbf{k}}^{2}
$$

where

$$
\mathbf{v}_{s}=\frac{e \overline{\mathbf{A}}}{m c}
$$

and $E_{\mathbf{k}}=\left[\left(\xi_{\mathbf{k}}+\left(e^{2} / 2 m c^{2}\right) \int\left(d^{2} \mathbf{p} /(2 \pi)^{2}\right) A_{\mathbf{p}}^{2}\right)^{2}+\Delta_{\mathbf{k}}^{2}\right]^{1 / 2}$. In Eq. (14), we have explicitly separated the contributions to the free energy from the homogeneous $(\mathbf{q}=0)$ superflow and those corresponding to $\mathbf{q} \neq 0$ components.

$\mathcal{Z}$ in Eq. (14) can be written as

$$
\begin{gathered}
\mathcal{Z}=\mathcal{Z}_{0} \exp \left\{\sum_{n} \operatorname{tr} \ln \widehat{Q}^{(1)}\left(i \omega_{n}\right)\right\} \exp \left\{\sum_{n} \operatorname{tr} \ln \left[1-\widehat{\mathcal{G}}\left(i \omega_{n}\right) \widehat{Q}^{(2)}\right]\right\}=\mathcal{Z}_{0} \cdot \widetilde{\mathcal{Z}}_{1} \cdot \widetilde{\mathcal{Z}}_{2}, \\
\widetilde{\mathcal{Z}}_{1}=\exp \left\{\sum_{n} \operatorname{tr} \ln \widehat{Q}^{(1)}\left(i \omega_{n}\right)\right\}=\prod_{\mathbf{k}}\left\{1+e^{-\left(\mathbf{k} \cdot \mathbf{v}_{s}+E_{k}\right) / T}\right\} \cdot \prod_{\mathbf{k}}\left\{1+e^{-\left(\mathbf{k} \cdot \mathbf{v}_{s}-E_{k}\right) / T}\right\} .
\end{gathered}
$$

$\widetilde{\mathcal{Z}}_{2}$ may now be calculated by expanding in powers of $\mathbf{A}_{\mathbf{q} \neq \mathbf{0}}$. To second order, we have

$$
\begin{aligned}
\ln \widetilde{\mathcal{Z}}_{2}= & \sum_{n} \operatorname{tr} \ln \left(1-\widehat{\mathcal{G}}\left(i \omega_{n}\right) \widehat{Q}^{(2)}\right) \simeq \sum_{n} \operatorname{tr}\left[-\widehat{\mathcal{G}}\left(i \omega_{n}\right) \widehat{Q}^{(2)}-\frac{1}{2}\left(-\widehat{\mathcal{G}}\left(i \omega_{n}\right) \widehat{Q}^{(2)}\right)^{2}\right] \\
= & -\frac{e^{2}}{2 m^{2} c^{2}} \sum_{n} \int \frac{d^{2} \mathbf{k}}{(2 \pi)^{2}} \int \frac{d^{2} \mathbf{q}}{(2 \pi)^{2}}\left\{\mathcal{G}_{11}\left(i \omega_{n}, \mathbf{k}_{+}\right) \mathcal{G}_{11}\left(i \omega_{n}, \mathbf{k}_{-}\right)\left(\mathbf{k}_{-} \cdot \mathbf{A}_{\mathbf{q}}\right)\left(\mathbf{k}_{+} \cdot \mathbf{A}_{-\mathbf{q}}\right)\right. \\
& +\mathcal{G}_{22}\left(i \omega_{n}, \mathbf{k}_{+}\right) \mathcal{G}_{22}\left(i \omega_{n}, \mathbf{k}_{-}\right)\left(\mathbf{k}_{+} \cdot \mathbf{A}_{\mathbf{q}}\right)\left(\mathbf{k}_{-} \cdot \mathbf{A}_{-\mathbf{q}}\right)+\mathcal{G}_{12}\left(i \omega_{n}, \mathbf{k}_{+}\right) \mathcal{G}_{21}\left(i \omega_{n}, \mathbf{k}_{-}\right)\left(\mathbf{k}_{+} \cdot \mathbf{A}_{\mathbf{q}}\right)\left(\mathbf{k}_{+} \cdot \mathbf{A}_{-\mathbf{q}}\right) \\
& \left.+\mathcal{G}_{12}\left(i \omega_{n}, \mathbf{k}_{+}\right) \mathcal{G}_{21}\left(i \omega_{n}, \mathbf{k}_{-}\right)\left(\mathbf{k}_{-} \cdot \mathbf{A}_{\mathbf{q}}\right)\left(\mathbf{k}_{-} \cdot \mathbf{A}_{-\mathbf{q}}\right)\right\}
\end{aligned}
$$

where $\mathbf{k}_{ \pm}=\mathbf{k} \pm \mathbf{q} / 2$. Note that only the paired electrons, i.e., the electrons near the Fermi surface, can produce a diamagnetic effect. Thus we can replace $\mathbf{k}$ that couples to $\mathbf{A}_{\mathbf{q}}$ and $\mathbf{v}_{s}$ by $\mathbf{k}_{F}$. Adopting the London gauge $\mathbf{q} \cdot \mathbf{A}_{\mathbf{q}}=0$, we simplify $\ln \widetilde{\mathcal{Z}}_{2}$ in Eq. (22) as

$$
\ln \widetilde{\mathcal{Z}}_{2} \simeq-\frac{e^{2}}{m^{2} c^{2}} \sum_{n} \int \frac{d^{2} \mathbf{k}}{(2 \pi)^{2}} \int \frac{d^{2} \mathbf{q}}{(2 \pi)^{2}} \frac{\left(i \omega_{n}+\mathbf{k}_{F} \cdot \mathbf{v}_{s}\right)^{2}+\xi_{+} \xi_{-}+\Delta_{+} \Delta_{-}}{W_{+} W_{-}}\left(\mathbf{k}_{F} \cdot \mathbf{A}_{\mathbf{q}}\right)\left(\mathbf{k}_{F} \cdot \mathbf{A}_{-\mathbf{q}}\right),
$$

where $\xi_{ \pm}=\xi_{\mathbf{k}_{ \pm}}, \Delta_{ \pm}=\Delta_{\mathbf{k}_{ \pm}}$and $W_{ \pm}=W_{\mathbf{k}_{ \pm}}$.

\section{FREE ENERGY, SUPERCURRENT AND RESPONSE FUNCTION}

The total free energy density of the superconductor in the Meissner state $\mathcal{F}$ is now given by the sum of contributions from both electrons and magnetic field, where the former can be calculated in terms of the partition function. We obtain

$$
\mathcal{F}=-T \ln \widehat{\mathcal{Z}}+\frac{1}{8 \pi} \int d^{2} \mathbf{r}|\nabla \times \mathbf{A}(\mathbf{r})|^{2}=\mathcal{F}_{1}+\mathcal{F}_{2},
$$

where

$$
\begin{aligned}
& \mathcal{F}_{1}=\frac{1}{2} n m v_{s}^{2}-T \int \frac{d^{2} \mathbf{k}}{(2 \pi)^{2}}\left\{\ln \left[1+e^{-\left(\mathbf{k}_{\mathrm{F}} \cdot \mathbf{v}_{s}+E_{k}\right) / T}\right]+\ln \left[1+e^{-\left(\mathbf{k}_{\mathrm{F}} \cdot \mathbf{v}_{s}-E_{k}\right) / T}\right]\right\}+\frac{n \Delta_{0}^{2}}{V_{0}} \\
& \mathcal{F}_{2}=\frac{1}{2 c} \int \frac{d^{2} \mathbf{q}}{(2 \pi)^{2}} \mathcal{K}\left(\mathbf{q}, \mathbf{v}_{s}, T\right) A_{\mathbf{q}}^{2}+\frac{1}{8 \pi} \int \frac{d^{2} \mathbf{q}}{(2 \pi)^{2}} q^{2} A_{\mathbf{q}}^{2}
\end{aligned}
$$

with 


$$
\mathcal{K}\left(\mathbf{q}, \mathbf{v}_{s}, T\right)=\frac{n e^{2}}{m c}\left(1+\frac{2 T}{n m} \sum_{n} \int \frac{d^{2} \mathbf{k}}{(2 \pi)^{2}}\left(\mathbf{k}_{\mathrm{F}}\right)_{\|}^{2} \frac{\left(i \omega_{n}+\mathbf{k}_{F} \cdot \mathbf{v}_{s}\right)^{2}+\xi_{+} \xi_{-}+\Delta_{+} \Delta_{-}}{W_{+} W_{-}}\right) .
$$

$\mathcal{F}_{1}$ describes the kinetic energy of the quasiparticles with energy spectrum $\pm E_{k}$ shifted by $\mathbf{k}_{F} \cdot \mathbf{v}_{s}$. Formally, it is nothing but the Doppler shift of the quasiparticle with proper momentum $\mathbf{k}$ in the lab frame with local superfluid velocity $\mathbf{v}_{s}(\mathbf{r})$. 24]

It is interesting to look at $\mathcal{F}_{2}$ in Eq. (26). In the linear limit $\mathbf{v}_{s} \rightarrow 0, \mathcal{K}\left(\mathbf{q}, \mathbf{v}_{s}, T\right)$ in Eq. (27) reduces to the usual linear response function of a superconductor in the Meissner state [25]. The fact that $\mathcal{K}\left(\mathbf{q}, \mathbf{v}_{s}, T\right)$ is indeed a response function can be seen from minimization of the free energy with respect to $\mathbf{A}_{\mathbf{q}}, \partial \mathcal{F}_{2} / \partial \mathbf{A}_{\mathbf{q}}=0$, which gives the Fourier component of the current inside the superconductor,

$$
\mathbf{j}_{\mathrm{SC}}(\mathbf{q})=-\mathcal{K}\left(\mathbf{q}, \mathbf{v}_{s}, T\right) \mathbf{A}_{\mathbf{q}}, \quad \text { for } \mathbf{q} \neq 0 .
$$

Therefore Eqs. (24)-(27) describe the Bogoliubov quasiparticles with the Doppler-shifted energy spectrum responding to a weakly spatially varying magnetic field. Note that $\mathcal{K}\left(\mathbf{q}, \mathbf{v}_{s}, T\right)$ is obtained in an infinite sample. In the presence of a surface boundary, an external current $\mathbf{j}_{\text {ext }}(\mathbf{q})$ is introduced to coincide with the real boundary condition (see discussion in Sec. IV), leading to the total current as

$$
\mathbf{j}_{\text {tot }}(\mathbf{q})=\frac{c}{4 \pi} q^{2} \mathbf{A}_{\mathbf{q}}=\mathbf{j}_{\text {ext }}(\mathbf{q})-\mathcal{K}\left(\mathbf{q}, \mathbf{v}_{s}, T\right) \mathbf{A}_{\mathbf{q}} .
$$

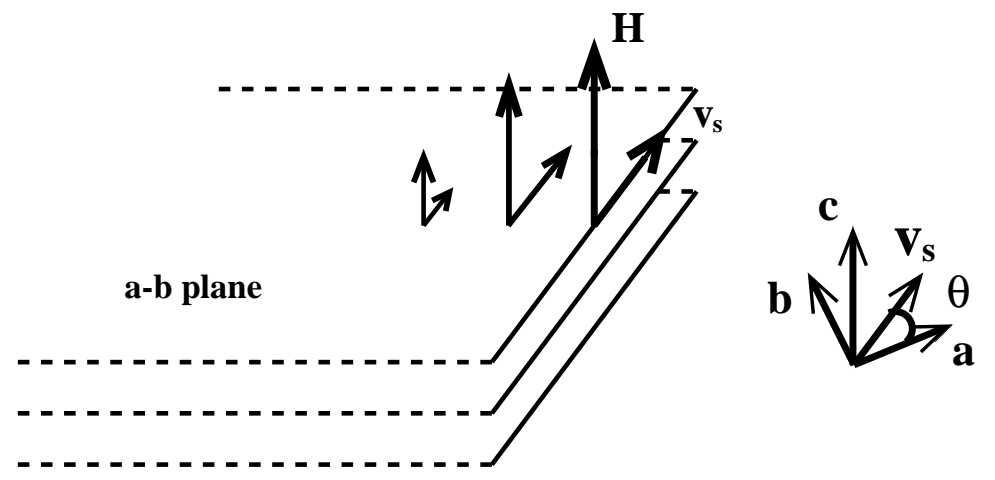

FIG. 1. Geometry of (010) surface case.

By summing over the Matsubara frequencies, Eq. (27) becomes

$$
\begin{gathered}
\mathcal{K}\left(\mathbf{q}, \mathbf{v}_{s}, T\right)=\frac{c}{4 \pi \lambda_{0}^{2}}+\delta \mathcal{K}\left(\mathbf{q}, \mathbf{v}_{s}, T\right), \\
\delta \mathcal{K}\left(\mathbf{q}, \mathbf{v}_{s}, T\right)=-2 \frac{c}{4 \pi \lambda_{0}^{2}}\left\langle\left(\hat{k}_{F}\right)_{\|}^{2} \int_{0}^{\infty} d \omega \operatorname{Re} \frac{\left[f\left(\omega-\mathbf{v}_{s} \cdot \mathbf{k}_{F}\right)-f\left(-\omega-\mathbf{v}_{s} \cdot \mathbf{k}_{F}\right)\right] \Delta_{\mathbf{k}}^{2}}{\sqrt{\omega^{2}-\Delta_{\mathbf{k}}^{2}}\left[\Delta_{k}^{2}+\left(\mathbf{q} \cdot \mathbf{k}_{F} /(2 m)\right)^{2}-\omega^{2}\right]}\right\rangle_{\mathrm{FS}} \\
=-\frac{c}{4 \pi \lambda_{0}^{2}}\left\{1-2\left\langle\left(\hat{k}_{F}\right)_{\|}^{2} \frac{\sinh ^{-1}\left(\frac{\mathbf{q} \cdot \mathbf{k}_{F}}{2 m \Delta_{\mathbf{k}}}\right)}{\left[\mathbf{q} \cdot \mathbf{k}_{F} /\left(2 m \Delta_{\mathbf{k}}\right)\right] \sqrt{1+\left[\mathbf{q} \cdot \mathbf{k}_{F} /\left(2 m \Delta_{\mathbf{k}}\right)\right]^{2}}}\right\rangle_{\mathrm{FS}}\right. \\
\left.+2\left\langle\left(\hat{k}_{F}\right)_{\|}^{2} \int_{0}^{\infty} d \omega \operatorname{Re} \frac{\left[f\left(\omega-\mathbf{k}_{F} \cdot \mathbf{v}_{s}\right)+f\left(\omega+\mathbf{k}_{F} \cdot \mathbf{v}_{s}\right)\right] \Delta_{\mathbf{k}}^{2}}{\sqrt{\omega^{2}-\Delta_{\mathbf{k}}^{2}}\left\{\Delta_{\mathbf{k}}^{2}+\left[\left(\mathbf{q} \cdot \mathbf{k}_{F}\right) /(2 m)\right]^{2}-\omega^{2}\right\}}\right\rangle_{\mathrm{FS}}\right\}
\end{gathered}
$$

where $f(x)$ is the Fermi function. $\delta \mathcal{K}\left(\mathbf{q}, \mathbf{v}_{s}, T\right)$ is geometry dependent. The first two terms in Eq. 32) represent the nonlocal renormalization of the $T=0$ response, while the third represents the combined nonlocal and nonlinear corrections to the $T$ dependence. For a real quasi-2D system, like the high- $T_{c}$ compounds, with the $a b$ plane as the conduction plane, we first consider the geometry where the magnetic field is parallel to the $\hat{c}$ axis and thus $\mathbf{v}_{s}$ and the penetration direction $\mathbf{q}$ are in the $a b$ plane, and in general, $\mathbf{v}_{s}$ makes an angle $\theta$ with the $\hat{a}$ axis. This geometry 
is shown in Fig. 1. Note that for $\mathbf{v}_{s}, \lambda$ antinode the possibility of the formation of an Andreev bound state exists. Walter et al. 27] have argued that such states can have significant effects on the low- $T$ penetration depth for $\mathbf{v}_{s} \|$ node. The effects on the field dependence of the penetration depth are beyond the scope of the current formalism, so results for these directions should be treated with caution. We will consider a different situation, where the magnetic field is in the $a b$ plane, at the end of Sec. IV.

An examination of Eq. (32) shows that the small regions around the four nodes give the main contribution when $v_{s} k_{F}, T \ll \Delta_{0}$. This reflects the low-energy quasiparticle excitations near nodes. It is straightforward to integrate out the angle of the Fermi wave vector, leading to

$$
\delta \mathcal{K}\left(\mathbf{q}, \mathbf{v}_{s}, T\right)=-\frac{c}{8 \pi \lambda_{0}^{2}} \frac{T}{\Delta_{0}} \sum_{l= \pm 1} u_{\theta l}^{2} F_{\lambda}\left(\frac{\alpha u_{\theta,-l}}{T}, \frac{\varepsilon u_{\theta l}}{T}\right),
$$

where $\alpha=q k_{F} /(2 \sqrt{2} m), \varepsilon=v_{s} k_{F} / \sqrt{2}, u_{\theta l}=|\cos \theta+l \sin \theta|$, and $F_{\lambda}\left(z_{1}, z_{2}\right)$ is a two-parameter scaling function

$$
F_{\lambda}\left(z_{1}, z_{2}\right)=\frac{\pi}{4} z_{1}+\left[\ln \left(e^{z_{2}}+1\right)+\ln \left(e^{-z_{2}}+1\right)\right]-\int_{0}^{z_{1}} d x\left[\frac{1}{e^{x-z_{2}}+1}+\frac{1}{e^{x+z_{2}}+1}\right]\left[1-\left(\frac{x}{z_{1}}\right)^{2}\right]^{1 / 2} .
$$

Now we may study two limiting cases.

$$
\text { A. } v_{s} k_{F} \ll T
$$

In this case we can expand $\mathcal{F}_{1}$ in Eq. (25) up to the leading order of $v_{s} k_{F} / T$

$$
\mathcal{F}_{1} \simeq \mathcal{F}_{\mathrm{BCS}}^{(0)}+\left[\frac{1}{2} n m v_{s}^{2}-\frac{1}{T} \int \frac{d^{2} \mathbf{k}}{(2 \pi)^{2}}\left(\mathbf{k}_{F} \cdot \mathbf{v}_{s}\right)^{2} \frac{e^{E_{\mathbf{k}}^{(0)} / T}}{\left(1+e^{E_{\mathbf{k}}^{(0)} / T}\right)^{2}}\right],
$$

where $E_{k}^{(0)}=\left(\xi_{k}^{2}+\Delta_{k}^{2}\right)^{1 / 2}$ and $\mathcal{F}_{\mathrm{BCS}}^{(0)}$ is the free energy density of the superconductor in the absence of a magnetic field,

$$
\mathcal{F}_{\mathrm{BCS}}^{(0)}=-T \int \frac{d^{2} \mathbf{k}}{(2 \pi)^{2}}\left[\ln \left(1+e^{-E_{k}^{(0)} / T}\right)+\ln \left(1+e^{E_{k}^{(0)} / T}\right)\right]+\frac{n \Delta_{0}^{2}}{V_{0}} .
$$

Inserting Eq. (19) into Eq. (35) yields

$$
\mathcal{F}_{1} \simeq \mathcal{F}_{\mathrm{BCS}}^{(0)}+\frac{1}{2 c} \mathcal{K}(0,0, T) A_{0}^{2},
$$

with $\mathcal{K}(0,0, T)=\frac{c}{4 \pi \lambda_{0}^{2}}+\delta \mathcal{K}(0,0, T)$ and

$$
\delta \mathcal{K}(0,0, T)=-\frac{c}{4 \pi \lambda_{0}^{2}}(2 \ln 2) \frac{T}{\Delta_{0}} .
$$

As for $\mathcal{F}_{2}$ in Eq. (26), up to the leading order of $\mathbf{A}_{\mathbf{q}}$, the response function $\mathcal{K}\left(\mathbf{q}, \mathbf{v}_{s}, T\right)$ is simply replaced by $\mathcal{K}(\mathbf{q}, 0, T)$, the usual nonlocal response function [25, 1 . It is straightforward to see from Eqs. (33) and (34) that

$$
\begin{aligned}
\delta \mathcal{K}(\mathbf{q}, 0, T) & =\delta \mathcal{K}(0,0, T)-\frac{c}{8 \pi \lambda_{0}^{2}} \sum_{l= \pm 1} u_{\theta l}^{2}\left\{\frac{\pi}{4} \frac{\alpha u_{\theta,-l}}{\Delta_{0}}-\frac{1}{\Delta_{0}} \int_{0}^{\alpha u_{\theta,-l}} d x f(x)\left[1-\frac{x^{2}}{\alpha^{2} u_{\theta,-l}^{2}}\right]^{1 / 2}\right\} \\
& \simeq\left\{\begin{array}{ll}
-\frac{c}{4 \pi \lambda_{0}^{2}}(2 \ln 2) \frac{T}{\Delta_{0}}, & \alpha u_{\theta,-l} \ll T, \quad l= \pm 1 \\
-\frac{c}{8 \pi \lambda_{0}^{2}} \sum_{l= \pm 1} u_{\theta l}^{2}\left(\frac{\pi}{4} \frac{\alpha u_{\theta,-l}}{\Delta_{0}}+\frac{3}{2} \zeta(3) \frac{T^{3}}{\Delta_{0} \alpha^{2} u_{\theta,-l}^{2}}\right), & \alpha u_{\theta,-l} \gg T, \quad l= \pm 1
\end{array},\right.
\end{aligned}
$$

a special case of which, $\theta=0$, has been presented in Ref. [1]. In Eq. (40) $\zeta(3) \simeq 1.20$ is Riemann's zeta function.

Therefore the change of the free energy due to the presence of the magnetic field is

$$
\Delta \mathcal{F}=\mathcal{F}-\mathcal{F}_{\mathrm{BCS}}^{(0)}=\frac{1}{8 \pi} \int \frac{d^{2} \mathbf{q}}{(2 \pi)^{2}}\left[q^{2}+\frac{4 \pi}{c} \mathcal{K}^{(0)}(\mathbf{q}, 0, T)\right] A_{\mathbf{q}}^{2} .
$$


Comparing Eq. (41) with Eq. (2), we seem to find a sign discrepancy. However, notice that the free energy in Eq. (2) is calculated at a fixed external current and hence is the Gibbs free energy, whereas that in Eq. (41) is the Helmholtz free energy, $\Delta \mathcal{F}^{(\mathrm{Helm})}$, since a fixed $\mathbf{A}_{\mathbf{q}}$ is assumed in the calculation. It can be checked that these two kinds of free energy are related to each other through the Legendre transformation

$$
\Delta \mathcal{F}^{(\mathrm{SD})}=\Delta \mathcal{F}^{(\mathrm{Helm})}-\frac{1}{c} \int \frac{d^{2} \mathbf{q}}{(2 \pi)^{2}} \mathbf{j}_{\mathrm{ext}}(\mathbf{q}) \cdot \mathbf{A}_{\mathbf{q}}=-\frac{1}{8 \pi} \int \frac{d^{2} \mathbf{q}}{(2 \pi)^{2}}\left[q^{2}+\frac{4 \pi}{c} \mathcal{K}^{(0)}(q, 0, T)\right] A_{\mathbf{q}}^{2} .
$$

In the last equality, Eq. (29) was used.

The entropy can be calculated from the Helmholtz free energy in Eq. (41) with A fixed, which is easily shown to be identical with that in Eq. (3)

$$
S(T)=-\left.\frac{\partial \Delta \mathcal{F}^{(\text {Helm })}}{\partial T}\right|_{\text {fixed } \mathbf{A}}=S^{(S D)}(T) .
$$

Equations (41) and (38) imply that in the local limit $\alpha \rightarrow 0, \Delta \mathcal{F} \propto T$. If this linear $T$ behavior were to hold at $T \rightarrow 0$, the third law of thermodynamics, the Nernst's theorem, would be violated as pointed out by Schopohl and Dolgov [10]. However, the intrinsic nonlocal effects may cut off the linear $T$ term [4] 17]. Kosztin and Leggett showed, in the special geometry where $\theta=0$, that the linear $T$ behavior of $\delta \mathcal{K}\left(\mathbf{q}, \mathbf{v}_{s} \rightarrow 0, T\right)$ will be changed to a higher power of $T$ when $T$ is smaller than $\alpha$. In the case of more general $\theta$, the linear $T$ term is preserved until $T$ is smaller than both $\alpha u_{\theta l}, l= \pm 1$. For $\mathbf{v}_{s}$ along any node, $u_{\theta l}=0$ for $l=1$ or -1 . The nonlocal effects thus disappear along this nodal direction. In this case, the linear $T$ singularity may be cut off by another effect, the nonlinear effect, which we now discuss.

$$
\text { B. } v_{s} k_{F} \gg T
$$

Notice that in Eq. (33), $\varepsilon$ is always accompanied by $u_{\theta l}$, which, for $\theta$ near any node, may be very small. We consider first the situation where $\theta$ is not close to any node, so that $\varepsilon u_{\theta l} \gg T$. The small perturbation parameter is now $T /\left(v_{s} k_{F} u_{\theta l}\right)$. The kinetic free energy $\mathcal{F}_{1}$ becomes

$$
\mathcal{F}_{1}=\frac{1}{2} n m v_{s}^{2}-2 T \int_{-\infty}^{\infty} d x N(x) \ln \left[1+e^{-x / T}\right]-\int \frac{d^{2} \mathbf{k}}{(2 \pi)^{2}} E_{\mathbf{k}}
$$

where

$$
N(x)=\int \frac{d^{2} \mathbf{k}}{(2 \pi)^{2}} \delta\left[x-\left(\mathbf{v}_{s} \cdot \mathbf{k}_{F}+E_{\mathbf{k}}\right)\right]=\frac{N_{0}}{2 \Delta_{0}} \sum_{l= \pm 1}\left(x+\varepsilon u_{\theta l}\right) \vartheta\left(x+\varepsilon u_{\theta l}\right)
$$

with $\vartheta(y)$ the step function and $N_{0}$ the density of states on the Fermi surface in the normal state. Inserting Eq. (45) into Eq. (44) we get

$$
\mathcal{F}_{1}=\mathcal{F}_{\mathrm{BCS}}^{(0)}+\frac{1}{2} n m v_{s}^{2}-\frac{1}{3} \frac{\zeta_{\theta}}{\sqrt{2}} \frac{n m k_{F}}{2 \Delta_{0}} v_{s}^{3}-\frac{\pi^{2}}{6} \frac{n m}{\Delta_{0} k_{F}} \sum_{l= \pm 1} u_{\theta l} v_{s} T^{2}
$$

where

$$
\zeta_{\theta}=\frac{1}{2} \sum_{l= \pm 1} u_{\theta l}^{3}=\frac{1}{2}\left(|\cos \theta+\sin \theta|^{3}+|\cos \theta-\sin \theta|^{3}\right) .
$$

The qualitative behavior of $\mathcal{F}_{1}$ as a function of both, $v_{s}$ and $T$, is consistent with that obtained by Volovik [11]. Here we see that the prefactors of both the $v_{s}^{3}$ and $v_{s} T^{2}$ terms are $\theta$ dependent.

The scaling function $F_{\lambda}\left(z_{1}, z_{2}\right)$ in Eq. (34) has the following asymptotic behavior at $z_{2} \gg 1$ :

$$
F_{\lambda}\left(z_{1}, z_{2}\right) \simeq\left\{\begin{array}{ll}
z_{2} & z_{1} \ll z_{2} \\
\frac{\pi z_{1}}{4}+\frac{z_{2}\left(z_{2}^{2}+\pi^{2}\right)}{6 z_{1}^{2}} & z_{2} \ll z_{1}
\end{array} .\right.
$$

This may be inserted into (33) to find that $\delta \mathcal{K}\left(\mathbf{q}, \mathbf{v}_{s}, T\right)=\sum_{l= \pm 1} \delta \mathcal{K}^{(l)}\left(\mathbf{q}, \mathbf{v}_{s}, T\right)$, where 


$$
\delta \mathcal{K}^{(l)}\left(\mathbf{q}, \mathbf{v}_{s}, T\right)= \begin{cases}-\frac{c}{8 \pi \lambda_{0}^{2}} u_{\theta l}^{3} \frac{\varepsilon}{\Delta_{0}}+\mathcal{O}\left(T e^{-\varepsilon / T}\right), & \alpha u_{\theta,-l} \ll \varepsilon u_{\theta l}, \\ -\frac{c}{8 \pi \lambda_{0}^{2}} u_{\theta l}^{2}\left[\frac{u_{\theta,-l} \pi}{4} \frac{\alpha}{\Delta_{0}}+\frac{u_{\theta l}^{3}}{6 u_{\theta,-l}^{2}} \frac{\varepsilon^{3}}{\alpha^{2} \Delta_{0}}+\frac{\pi^{2} u_{\theta l}}{6 u_{\theta,-l}^{2}} \frac{\varepsilon T^{2}}{\alpha^{2} \Delta_{0}}\right], & \alpha u_{\theta,-l} \gg \varepsilon u_{\theta l} .\end{cases}
$$

It is clear from Eqs. (40) and (49), that the linear $T$ term in $\delta \mathcal{K}\left(\mathbf{q}, \mathbf{v}_{s}, T\right)$, and hence in $\mathcal{F}_{2}$, is modified to a higher power of $T$ by either nonlinear or nonlocal effects. Combining this result with $\mathcal{F}_{1}$ in Eq. (44), we see that a thermodynamic instability, associated with the violation of the Nernst theorem, is avoided.

For $\theta$ near a nodal value, $u_{\theta l} \simeq 0$ for $l=1$ or -1 . This means that those quasiparticles in nodal regions with wavevectors nearly perpendicular to $\mathbf{v}_{s}$ contribute negligibly to the nonlinear effect such that the linear $T$ singularity in the free energy may not be cut off. These quasiparticles, however, acquire large nonlocal effects since $\mathbf{q} \perp \mathbf{v}_{s}$, which may effectively modify the $T$ dependence of the free energy. Similarly, it follows that the quasiparticles which generate a small nonlocal effect produce much larger nonlinear effects.

With the expression for the free energy, we can easily obtain the supercurrent. The part of the supercurrent arising from the kinetic free energy $\mathcal{F}_{1}$ in Eq. (46) is given by

$$
\mathbf{j}_{s c}^{(k i n)}=-\frac{e}{m} \frac{\partial \mathcal{F}_{1}}{\partial \mathbf{v}_{s}}=-e n \mathbf{v}_{s}+e n \frac{v_{s} k_{F}}{2 \Delta_{0}}\left(\frac{\zeta_{\theta}}{\sqrt{2}} \mathbf{v}_{s}+\frac{1}{3 \sqrt{2}} \frac{\partial \zeta_{\theta}}{\partial \theta} \hat{z} \times \mathbf{v}_{s}\right),
$$

where $\hat{z} \| \hat{c}$. It is clear that due to the nonlinear correction $\mathbf{j}_{s c}^{(k i n)}$ is not parallel to $\mathbf{v}_{s}$ except for $\mathbf{v}_{s} \|$ node or antinode in the case of which we recover the Yip-Sauls nonlinear supercurrent form [12, 13,

$$
\mathbf{j}_{s c}^{(k i n)}=\left\{\begin{array}{lc}
-e n \mathbf{v}_{s}\left(1-\frac{v_{s} k_{F}}{2 \Delta_{0}}\right), & \mathbf{v}_{s} \| \text { node } \\
-e n \mathbf{v}_{s}\left(1-\frac{1}{\sqrt{2}} \frac{v_{s} k_{F}}{2 \Delta_{0}}\right), & \mathbf{v}_{s} \| \text { antinode }
\end{array}\right.
$$

The fact that $\mathbf{j}_{s c}^{(k i n)}$ is not parallel to $\mathbf{v}_{s}$ for general $\theta$ leads to the interesting magnetic torque phenomenon that has been discussed in Ref. [13].

$\mathbf{j}(\mathbf{q})$ can be obtained from Eqs. (28), (30), and (49). It is nontrivial to examine the supercurrent in the local limit

$$
\mathbf{j}(\mathbf{q} \rightarrow 0)=\left\{\begin{array}{lc}
-\frac{c}{4 \pi \lambda_{0}^{2}}\left(1-\frac{v_{s} k_{F}}{\Delta_{0}}\right) \mathbf{A}_{\mathbf{q} \rightarrow 0}, & \mathbf{v}_{s} \| \text { node } \\
-\frac{c}{4 \pi \lambda_{0}^{2}}\left(1-\frac{1}{\sqrt{2}} \frac{v_{s} k_{F}}{\Delta_{0}}\right) \mathbf{A}_{\mathbf{q} \rightarrow 0}, & \mathbf{v}_{s} \| \text { antinode }
\end{array}\right.
$$

If we simply take $(e / m c) \mathbf{A}_{\mathbf{q} \rightarrow 0}$ as $\mathbf{v}_{s}$, we find that $\mathbf{j}(\mathbf{q} \rightarrow 0)$ is of the same structure as in $\mathbf{j}_{s c}^{(k i n)}$ in Eq. (51), but with an extra prefactor 2 in the nonlinear correction term. Note that $\mathbf{j}(\mathbf{q}=0)$ in the present theory is not equivalent to $\mathbf{j}_{s c}^{(k i n)}$, but instead,

$$
\mathbf{j}(\mathbf{q}=0)=-\frac{e}{m} \frac{\partial \mathcal{F}}{\partial \mathbf{v}_{s}}=\mathbf{j}_{s c}^{(k i n)}-\frac{e}{2 m c} \int \frac{d^{2} \mathbf{q}}{(2 \pi)^{2}} \frac{\partial \mathcal{K}\left(\mathbf{q}, \mathbf{v}_{s}, T\right)}{\partial \mathbf{v}_{s}} A_{q}^{2}
$$

If $\mathcal{K}\left(\mathbf{q}, \mathbf{v}_{s}, T\right)$ can be replaced by its local-limit value, Eq. (53) becomes

$$
\mathbf{j}(\mathbf{q}=0) \simeq \begin{cases}-\frac{c}{4 \pi \lambda_{0}^{2}}\left(1-\frac{v_{s} k_{F}}{\Delta_{0}}\right) \frac{m c}{e} \mathbf{v}_{s}, & \mathbf{v}_{s} \| \text { node } \\ -\frac{c}{4 \pi \lambda_{0}^{2}}\left(1-\frac{1}{\sqrt{2}} \frac{v_{s} k_{F}}{\Delta_{0}}\right) \frac{m c}{e} \mathbf{v}_{s}, & \mathbf{v}_{s} \| \text { antinode }\end{cases}
$$

We conclude that the nonlinear correction we have obtained from the $\mathbf{q} \rightarrow 0$ mode is formally two times that in Yip-Sauls theory. The reason for this discrepency is that we treat the response of the superconductor to $\mathbf{A}_{\mathbf{q} \neq 0}$ modes perturbatively, while the nonlinear term $\left(v_{s}^{3}\right.$ term in the free energy) coming from the Doppler shift is not a perturbative result. To see this clearly, we make a naive perturbation calculation of the response of the superconductor to a constant $v_{s}$. The partition function is (Compare with Eq. 14)

$$
\mathcal{Z}=\exp \left\{\sum_{n} \operatorname{Tr} \ln \left[\widehat{P}^{(1)}\left(i \omega_{n}\right)+\widehat{P}^{(2)}\right]\right\}=\exp \left\{\sum_{n} \operatorname{Tr} \ln \left[\widehat{P}^{(1)}\left(i \omega_{n}\right)\right]\right\} \exp \left\{\sum_{n} \operatorname{Tr} \ln \left[1+\widehat{P}^{(1)-1}\left(i \omega_{n}\right) \widehat{P}^{(2)}\right]\right\}
$$

where

$$
\widehat{P}^{(1)}\left(i \omega_{n}\right)=\left(\begin{array}{cc}
-i \omega_{n}+\xi_{\mathbf{k}} & \Delta_{\mathbf{k}} \\
\Delta_{\mathbf{k}} & -i \omega_{n}-\xi_{\mathbf{k}}
\end{array}\right), \quad \widehat{P}^{(2)}=\mathbf{k}_{F} \cdot \mathbf{v}_{s}\left(\begin{array}{ll}
1 & 0 \\
0 & 1
\end{array}\right)
$$


with $\widehat{P}^{(2)}$ assumed small. The free energy is

$$
\mathcal{F}=-T \ln \mathcal{Z}=-T \sum_{n} \operatorname{Tr} \ln \left[\widehat{P}^{(1)}\left(i \omega_{n}\right)\right]-T \sum_{n} \operatorname{Tr} \ln \left[1+\widehat{P}^{(1)-1}\left(i \omega_{n}\right) \widehat{P}^{(2)}\right] .
$$

The $v_{s}^{3}$ term of present interest is expected to arise from

$$
-T \sum_{n} \operatorname{Tr}\left\{\frac{1}{3}\left[\widehat{P}^{(1)-1}\left(i \omega_{n}\right) \widehat{P}^{(2)}\right]^{3}\right\}=-\frac{1}{3} T \sum_{n} \operatorname{Tr}\left(\mathbf{v}_{s} \cdot \mathbf{k}_{F}\right)^{3}\left(\begin{array}{cc}
\frac{-i \omega_{n}-\xi_{\mathbf{k}}}{\left(i \omega_{n}\right)^{2}-\xi_{\mathbf{k}}^{2}-\Delta_{\mathbf{k}}^{2}} & \frac{-\Delta_{\mathbf{k}}}{\left(i \omega_{n}\right)^{2}-\xi_{\mathbf{k}}^{2}-\Delta_{\mathbf{k}}^{2}} \\
\frac{\Delta_{\mathbf{k}}}{\left(i \omega_{n}\right)^{2}-\xi_{\mathbf{k}}^{2}-\Delta_{\mathbf{k}}^{2}} & \frac{-i \omega_{n} \xi_{\xi_{\mathbf{k}}}}{\left(i \omega_{n}\right)^{2}-\xi_{\mathbf{k}}^{2}-\Delta_{\mathbf{k}}^{2}}
\end{array}\right)^{3}=0 .
$$

A nonlinear term $\propto v_{s}^{3}$ cannot be obtained from the perturbation theory, since the perturbation calculation implies $\mathbf{v}_{s} \cdot \mathbf{k}_{F} \ll \Delta_{\mathbf{k}}$, while the nonlinear $v_{s}^{3}$ term comes from those $\mathbf{k}$ points near nodes on the Fermi surface satisfying $\Delta_{\mathbf{k}}<\mathbf{v}_{s} \cdot \mathbf{k}_{F}$. Therefore the crucial step of taking $(e / m c) A_{\mathbf{q} \rightarrow 0}$ as $\mathbf{v}_{s}$ in obtaining Eq. (54) is an approximation since the couplings of the quasiparticles to $\mathbf{A}_{\mathbf{q} \neq 0}$ are treated perturbatively. Nevertheless we expect that the qualitative scaling behavior will be correctly reproduced with the present formalism. The renormalizations of the naive results to be expected from treating the magnetic field in a fully self-consistent manner are discussed in the Appendix.

\section{PENETRATION DEPTH}

The penetration depth for a half infinite system may be defined as

$$
\lambda=\frac{1}{H} \int_{0}^{\infty} H(y) d y,
$$

where $H$ is the magnetic field at the surface. A specular scattering surface boundary condition on the quasiparticle wave functions is assumed, which replaces the surface with a current sheet of the form

$$
\mathbf{j}_{\text {ext }}(y)=-\frac{c}{2 \pi} H \delta(y) \hat{x} .
$$

in an infinite system [26].

\section{A. Penetration depth at a constant $v_{s}$}

As a first step, in this subsection, we study the penetration depth in the system characterized by an electromagnetic response function calculated in the presence of a constant $v_{s}$. Since the true, self-consistently determined superfluid velocity decays with distance from the surface, this procedure introduces errors in the nonlinear terms, which we discuss below and estimate in some detail in the Appendix. For the moment, we consider Maxwell's Eq.

$$
\nabla^{2} \mathbf{A}=-\frac{4 \pi}{c}\left(\mathbf{j}_{e x t}+\mathbf{j}_{s c}\right)
$$

and the London Eq. (28), obtaining

$$
\lambda_{\text {spec }}=\frac{2}{\pi} \int_{0}^{\infty} \frac{d q}{4 \pi \mathcal{K}\left(\mathbf{q}, \mathbf{v}_{s}, T\right) / c+q^{2}} \simeq \lambda_{0}-\frac{8}{c} \int_{0}^{\infty} d q \frac{\delta \mathcal{K}\left(\mathbf{q}, \mathbf{v}_{s}, T\right)}{\left(\lambda_{0}^{-2}+q^{2}\right)^{2}} .
$$

Here $\mathbf{v}_{s}$ is now to be understood as its value at the surface $v_{s}=v_{s}(y=0)=e \lambda_{0} H / m c$ as given by the solution to the linear, local electrodynamics problem. Obviously, $\lambda_{\text {spec }}$ includes both the nonlocal and nonlinear effects. It is expected to reduce exactly to the nonlocal expression of Kosztin and Leggett [| $\mid$ if the $\mathbf{v}_{s}$ dependence is neglected, and (qualitatively) to the nonlinear expression of Yip and Sauls 12 if the $q$ dependence is neglected. We first go over these two limiting cases.

For the linear limit $v_{s} \rightarrow 0$, the qualitative behavior of the penetration depth depends on two effective nonlocal energy scales, $E_{\text {nonloc }}^{(+)}=v_{F} u_{\theta l_{1}} / \lambda_{0}$ and $E_{\text {nonloc }}^{(-)}=v_{F} u_{\theta l_{2}} / \lambda_{0}$ for $l_{1}, l_{2}= \pm 1$ and $u_{\theta l_{1}} \geq u_{\theta l_{2}}$. It is shown that

$$
\frac{\delta \lambda_{\text {spec }}^{(\operatorname{lin})}}{\lambda_{0}} \simeq \begin{cases}\frac{1}{2}(\ln 2) \sum_{l= \pm 1} u_{\theta l}^{2} \frac{T}{\Delta_{0}}, & \text { for } E_{\text {nonloc }}^{(+)}, E_{\text {nonloc }}^{(-)} \ll T \\ \frac{1}{2}(\ln 2) u_{\theta l_{1}}^{2} \frac{T}{\Delta_{0}}+\frac{\pi}{16 \sqrt{2}} \kappa^{-1} u_{\theta l_{1}} u_{\theta l_{2}}^{2}, & \text { for } E_{\text {nonloc }}^{(-)} T \ll E_{\text {nonloc }}^{(+)} \\ \frac{\pi}{16 \sqrt{2}} \kappa^{-1} \sum_{l= \pm 1} u_{\theta,-l} u_{\theta l}^{2}+0.80 \kappa\left(\frac{T}{\Delta_{0}}\right)^{2} \sum_{l= \pm 1} \frac{u_{\theta l}^{2}}{u_{\theta,-l}}, & \text { for } T \ll E_{\text {nonloc }}^{(+)}, E_{\text {nonloc }}^{(-)}\end{cases}
$$


For $\theta \sim 0, E_{\text {nonloc }}^{(-)} \simeq E_{\text {nonloc }}^{(+)}$and there is no intermediate parameter region $E_{\text {nonloc }}^{(-)} \ll T \ll E_{\text {nonloc }}^{(+)}$. In this case, we recover Kosztin and Leggett's result [ [⿴囗十). However, for $\theta$ near any node, $E_{\text {nonloc }}^{(-)}$disappears, and the linear $T$ behavior of the penetration depth is preserved even at $T \ll v_{F} / \lambda_{0}$, but with a reduced prefactor. We conclude that in the linear limit the nonlocal effects fail to cut off the linear $T$ dependence of the penetration depth when $v_{s}$ is along nodal directions. On the other hand, it is precisely in this limit that the effects of Andreev bound states on the penetration depth are expected to be largest. Although the influence of these states on the field dependence has not yet been calculated, it appears unlikely that the naive Yip-Sauls result can apply.

For the local limit $\mathbf{q} \rightarrow 0$, Eq. (62) becomes

$$
\lambda_{\text {spec }}^{(\text {loc })}=\left(\frac{c}{4 \pi \mathcal{K}\left(\mathbf{q} \rightarrow 0, \mathbf{v}_{s}, T\right)}\right)^{1 / 2}
$$

Again, for a general $\theta$, there are two effective nonlinear energy scales, $E_{\text {nonlin }}^{(+)}=v_{s} k_{F} u_{\theta l_{1}}$ and $E_{\text {nonlin }}^{(-)}=v_{s} k_{F} u_{\theta l_{2}}$. One gets

$$
\frac{\delta \lambda_{\text {spec }}^{(\text {loc })}}{\lambda_{0}} \simeq\left\{\begin{array}{ll}
\frac{1}{2}(\ln 2) \sum_{l= \pm 1} u_{\theta l}^{2} \frac{T}{\Delta_{0}}, & E_{\text {nonlin }}^{(+)}, E_{\text {nonlin }}^{(-)} \ll T \\
\frac{1}{2}(\ln 2) u_{\theta l_{2}}^{2} \frac{T}{\Delta_{0}}+\frac{u_{\theta l_{1}}^{3}}{2 \sqrt{2}} \frac{v_{s} k_{F}}{2 \Delta_{0}}, & E_{\text {nonlin }}^{(-)} \ll T \ll E_{\text {nonlin }}^{(+)} \\
\frac{\zeta \theta}{\sqrt{2}} \frac{v_{s} k_{F}}{2 \Delta_{0}}+\mathcal{O}\left(T e^{-v_{s} k_{F} / \sqrt{2} T}\right), & T \ll E_{\text {nonlin }}^{(+)}, E_{\text {nonlin }}^{(-)}
\end{array} .\right.
$$

Now we are in the position to study the penetration depth with both nonlocal and nonlinear effects. We are basically interested in the case of $T \ll v_{s} k_{F}$. Inserting Eqs. (33) and (48) into Eq. (62) leads to

$$
\frac{\delta \lambda_{\mathrm{spec}}}{\lambda_{0}}=\sum_{l= \pm 1} \frac{\delta \lambda_{\mathrm{spec}}^{(l)}}{\lambda_{0}}
$$

where

$$
\frac{\delta \lambda_{\text {spec }}^{(l)}}{\lambda_{0}} \simeq \frac{k_{F} u_{\theta,-l}^{3}}{8 \sqrt{2} \pi m^{3} \lambda_{0}^{3} \Delta_{0} v_{s}^{2}} \eta_{1}\left(h_{\theta l}\right)+\frac{T^{2} u_{\theta,-l}^{3}}{4 \sqrt{2} \pi m^{3} \lambda_{0}^{3} \Delta_{0} v_{s}^{4} k_{F} u_{\theta l}^{2}} \eta_{2}\left(h_{\theta l}\right)
$$

with

$$
\begin{aligned}
& \eta_{1}(x)=4 x^{3}\left[\frac{2 x}{1+4 x^{2}}+\arctan (2 x)\right]+\frac{x^{2}\left(3 \pi+16 x^{2}+96 x^{4}-48 x^{3}\left(1+4 x^{2}\right) \arctan (1 / 2 x)\right.}{6\left(1+4 x^{2}\right)}, \\
& \eta_{2}(x)=\frac{4 \pi^{2} x^{4}\left[2+12 x^{2}-6 x\left(1+4 x^{2}\right) \arctan (1 / 2 x)\right]}{3\left(1+4 x^{2}\right)}
\end{aligned}
$$

and

$$
h_{\theta l}=\frac{u_{\theta l}}{u_{\theta,-l}} h, \quad h=m \lambda_{0} v_{s}=\frac{3}{\pi} \kappa \frac{H}{H_{0}},
$$

defining the competition between the nonlinear and nonlocal effects, with $H_{0}$ as defined in Eq. (5). It is straightforward to get the asymptotic behavior of the penetration depth:

$$
\frac{\delta \lambda_{\text {spec }}^{(l)}}{\lambda_{0}} \simeq \frac{\pi}{4 \sqrt{2}} u_{\theta l}^{3} \kappa^{-1} h+c_{\theta l 1} \kappa^{-1} \frac{1}{h^{2}}+c_{\theta l 2} \kappa \frac{1}{h^{4}} \frac{T^{2}}{\Delta_{0}^{2}}, \quad h_{\theta l} \gg 1,
$$

where the nonlinear effect dominates, with $c_{\theta l 1}=0.0082 u_{\theta,-l}^{3}$ and $c_{\theta l 2}=0.0059 u_{\theta,-l}^{3} / u_{\theta l}^{2}$, and

$$
\frac{\delta \lambda_{\text {spec }}^{(l)}}{\lambda_{0}} \simeq \frac{\pi}{8 \sqrt{2}} d_{\theta l 1} \kappa^{-1}+d_{\theta l 2} \kappa^{-1} h^{2}+d_{\theta l 3} \kappa \frac{T^{2}}{\Delta_{0}^{2}}, \quad h_{\theta l} \ll 1,
$$

where the nonlocal effects dominate, with $d_{\theta l 1}=0.50 u_{\theta l}^{2} u_{\theta,-l}, d_{\theta l 2}=1.09 u_{\theta l}^{4} / u_{\theta,-l}$, and $d_{\theta l 3}=0.47 u_{\theta l}^{2} / u_{\theta,-l}$. In the local, $H \rightarrow 0$ limit, the result reduces to 


$$
\frac{\delta \lambda_{\text {spec }}}{\lambda_{0}} \simeq \frac{3}{2 \sqrt{2}} \zeta_{\theta} \frac{H}{H_{0}}
$$

which is apparently a factor of $3 / 2$ larger than the result (44). In fact, the comparison is a bit more subtle, as we have taken a definition of the penetration depth which differs slightly from that of Yip and Sauls. [12] This comparison is discussed further in the Appendix.

From Eqs. (70) and (71) one now sees clearly that the linear $H$ dependence of $\delta \lambda_{\text {spec }}^{(l)}$ at $h_{\theta l} \gg 1$ is modified to an $H^{2}$ dependence when $h_{\theta l} \ll 1$, implying that the nonlinear effect may be cutoff by the nonlocal effects. The crossover field for this to happen is defined by $h_{\theta l} \sim 1$. For most $\theta, u_{\theta l}$ and $u_{\theta,-l}$ are order of unity and the crossover field is simply defined by $h \sim 1$, i.e., $H^{*} \simeq \pi \kappa^{-1} H_{0} / 3 \simeq H_{c 1}$, the lower critical field. Since at $H \geq H_{c 1}$ the Meissner state is unstable to the Abrikosov vortex state, the nonlinear Meissner effect is effectively unobservable due to the nonlocal effect in this geometry 22]. In Fig. 2, we display the magnetic field dependence of the penetration depth for $\theta=0$. It is clear that in this case the predicted linear behavior in $H$ is only recovered well above $H_{c 1}$. On the other hand, for $\theta$ very close to a node, $\min \left(h_{\theta l}\right) \sim 0$, meaning that the crossover field can be so small that the nonlinear effect cannot be cut off by the nonlocal one [22]. The physical interpretation of this is that only those quasiparticle excitations near node regions can reduce the shielding current. When $\mathbf{v}_{s}$ is along a nodal direction, those quasiparticles near this node acquire a large nonlinear energy shift, but on the contrary a negligible nonlocal effect since $\mathbf{v}_{s}$ is perpendicular to $\mathbf{q}$. This result suggests that the best chance for experimentalists to see the nonlinear Meissner effect is in (110) surface geometry. We again caution, however, that for this geometry, we do not take account of the anomalous Meissner currents [27] carried by the Andreev surface bound state [28]. In Fig. 3 we show the $\theta$ dependence of the normalized penetration depth correction at $T=0$.

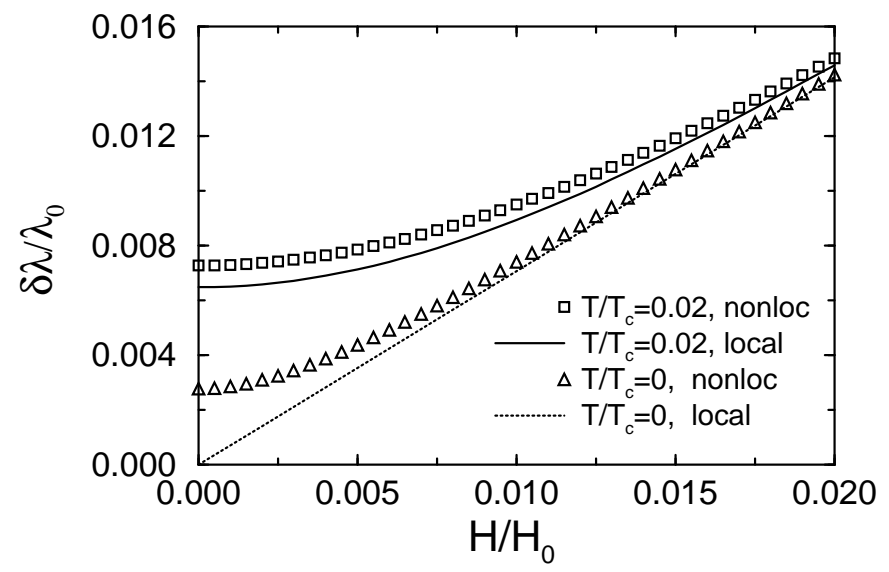

FIG. 2. Magnetic field dependence of the normalized penetration depth correction $\delta \lambda(H, T) / \lambda_{0}$ for $\theta=0 . \kappa=100$ is assumed and hence $H_{c 1} / H_{0}=0.01$.

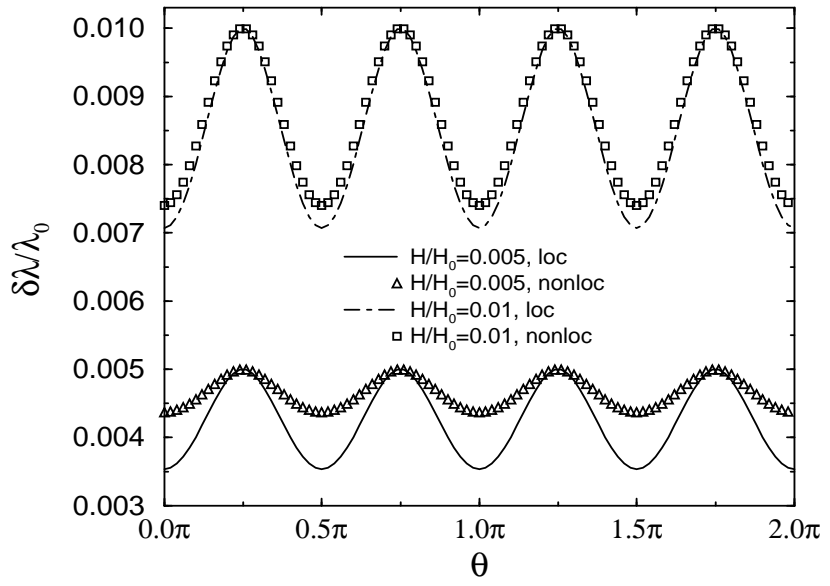


FIG. 3. $\theta$ dependence of the normalized penetration depth correction $\delta \lambda(H) / \lambda_{0}$ for $T=0 . \kappa$ is the same as in Fig. 2 .

Examining the $T$ dependence of $\delta \lambda$ in Eqs. (70) and (71) we find that for both $h_{\theta l} \gg 1$ and $\ll 1$ the leading $T$ term is quadratic. In Fig. 4(a), we plot the $T$ dependence of the penetration depth at fixed $H / H_{0}$ to show explicitly this feature. We also display $[\lambda(H, T)-\lambda(0, T)] / \lambda_{0}$ in Fig. 4(b) as a function of $T$ in comparison. Note in particular that the magnitude of the field dependence decreases with increasing temperature, as is to be expected for any effect which depends on the sharpness of the $d$-wave nodes. While the size of this decrease is diminished by the nonlocal corrections, there is never an increase in field dependence with increasing temperature, as observed in experiment (see discussion below).
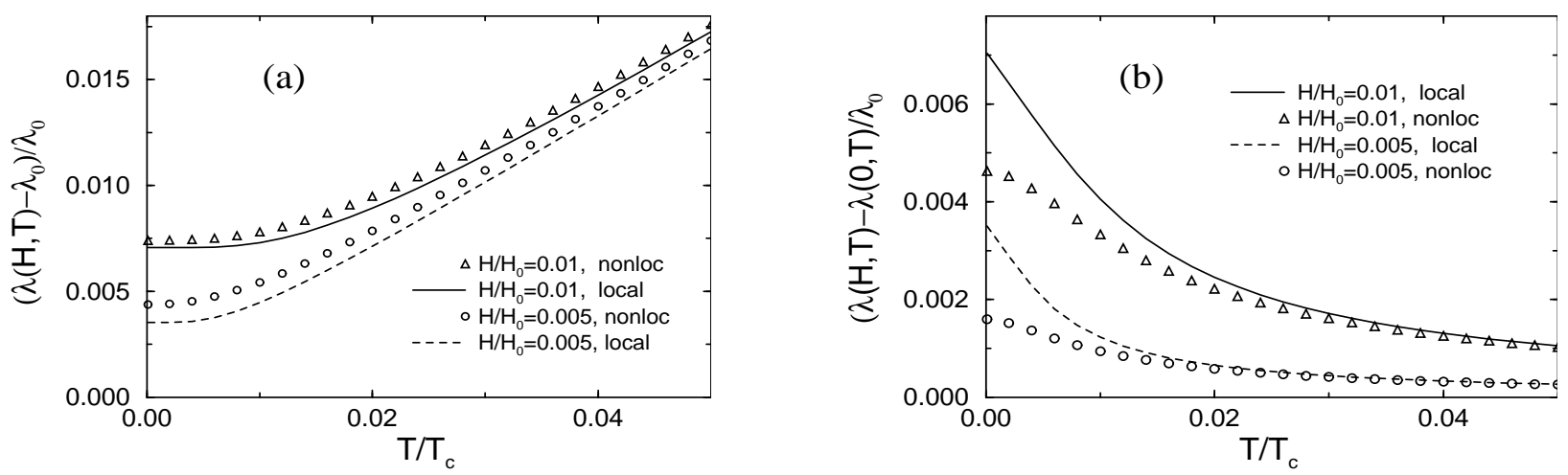

FIG. 4. $T$ dependence of (a) the normalized penetration depth correction $\delta \lambda(H, T) / \lambda_{0}$; (b) the normalized penetration depth correction $(\lambda(H, T)-\lambda(0, T)) / \lambda_{0} . \kappa$ is the same as in Fig. 2.

\section{B. Penetration depth in (001) surface case}

Up to now, we have discussed in detail the penetration depth when the field is along the $\hat{c}$-axis direction. In that case, all the interesting physics is in the $2 \mathrm{D} a b$ plane and it is implied that the possible interlayer transport kinetic energy is absorbed into the normal state energy spectrum. However, one may also consider the special situation in the quasi-2D cuprates in which the experimental configuration is characterized by a (001) surface with $\mathbf{H} \| a b$. In this case, $\mathbf{A}$ and $\mathbf{v}_{s}$ are still in the $a b$ plane (perpendicular to $H$ ) forming an angle $\theta$ with the $\hat{a}$ axis. The nonlinear effect that has been discussed in the previous sections remains the same. However, the normal of the surface, and hence the penetration direction $\mathbf{q}$, is perpendicular to the $a b$ plane. If the quasiparticles are confined rigorously to the $a b$ plane, they will not contribute to the nonlocal effects. But on the other hand, the magnetic field in this case is not screened $(\lambda \rightarrow \infty)$ [29]. In a real system, an interlayer coupling exists, leading to a nonvanishing $E_{\text {nonloc }}^{(a b)}$ [17]. Here we confine ourselves to the situation of coherent transport along the $\hat{c}$ direction. This may be a reasonable model at least for the YBCO material. To avoid confusing notation, we keep $\mathbf{k}$ as a $2 \mathrm{D}$ vector in the $a b$ plane, while we use $k_{c}$ as the momentum along the $\hat{c}$ direction. The energy spectrum of the quasiparticles in the superconducting state in the absence of the magnetic field is $E_{\mathbf{k}, k_{c}}^{\prime}=\sqrt{\xi_{\mathbf{k}, k_{c}}^{\prime 2}+\Delta_{\mathbf{k}}^{2}}$, where

$$
\xi_{\mathbf{k}, k_{c}}^{\prime}=\frac{k^{2}}{2 m}+\frac{k_{c}^{2}}{2 M}-\mu
$$

with $M \gg m$ the effective mass along the $\hat{c}$ direction. We have assumed that the pairing order parameter has no $k_{c}$ dependence. Now a parallel calculation for the penetration depth can be done. It is shown that the momentum $q$ picks out $k_{c}$ so as to replace the first argument of the scaling function $F_{\lambda}$ in Eq. (33) by $q k_{F c} /(2 M T)$. Thus the nonlocal energy scale becomes $E_{\text {nonloc }}^{(a b)} \simeq k_{F c} /\left(M \lambda_{0}\right) \simeq \xi_{0 c} \Delta_{0} / \lambda_{0}$ which is no longer $\theta$ dependent. One immediately sees that the main results presented for $H \| c$ case remain: the available nonlocal effects in (001) surface case serve to cut off the linear $T$ singularity and modify the $T$ dependence of the penetration depth to a quadratic one; at extremely low $T$, they cut off the linear $H$ dependence when the field is below a crossover critical field $H^{*(a b)}$ which is found to be $H^{*(a b)} \simeq\left(\xi_{0 c} / \xi_{0}\right) H^{*} . H^{*(a b)}$ is much smaller than $H^{*}$ since the $\hat{c}$-axis coherence length is $\xi_{0 c} \simeq 3 A$ as 
opposed to the in-plane coherence length of $\xi_{0} \simeq 15 \mathrm{~A}$. On the other hand, the lower critical field is also much smaller for this geometry, $H_{c 1}^{(a b)} \simeq \frac{\Phi_{0}}{4 \pi \lambda_{0} \lambda_{0 c}} \ln \bar{\kappa}$ [3], where $\lambda_{0 c}$ is the penetration depth for supercurrents along the $\hat{c}$ axis, and $\bar{\kappa}=\sqrt{\kappa \kappa_{0 c}}$ with $\kappa_{0 c}$ the $\hat{c}$-axis Ginzburg-Landau parameter. Using $\lambda_{0 c} \simeq 0.5-1 \times 10^{4} \mathrm{~A}$, we find a large crossover field $H^{*(a b)} \simeq H_{c 1}^{(a b)}$, making it still impossible from a practical point of view to extract a linear- $H$ term [31].

For highly anisotropic samples like BSCCO system, the above argument might not apply. A full treatment of this problem awaits a generally accepted theory of the (incoherent) $\hat{c}$-axis transport in the normal state. In addition, it is possible that surface-barrier effects render the field of first flux penetration much larger than we have estimated, leaving a large range of fields where the nonlocal effects can be neglected for this geometry. We discuss this situation below.

\section{DISCUSSION}

We first summarize our results. In this paper, we have investigated a clean (quasi-) $2 \mathrm{D} d_{x^{2}-y^{2}}$-wave superconductor in the Meissner state based on the weak-coupling BCS theory. The existence of nodes on the Fermi surface leads to several important effects at low energy that have nontrivial consequences on the free energy and the penetration depth. In addition to the thermal excitations of quasiparticles with typical energy scale $T$, there are also nonlocal effects, with energy scale $E_{\text {nonloc }}$, due to the divergent effective size of Cooper pairs along nodal directions, and nonlinear effects with energy scale $E_{\text {nonlin }}$, arising from the magnetic field-induced quasiparticle excitations. Taking the approximation of a slow varying supercurrent in space, we have formulated the Helmholtz free energy for the description of Bogoliubov quasiparticles with Doppler-shifted energy spectrum, and of the response of these quasiparticles to a weakly spatially varying magnetic field. The free energy is shown, after a Legendre transformation, to reduce to Schopohl-Dolgov's free energy at $E_{\text {nonlin }} \ll T$, and to Volovik's singular free energy form at $E_{\text {nonlin }} \gg T$. The resulting response function includes both nonlocal and nonlinear effects, from which a two-parameter scaling function of the penetration depth $\delta \lambda(H ; T) \simeq F\left(E_{\text {nonlin }} / T, E_{\text {nonloc }} / T\right)$ is obtained. The well-known linear $T$ dependence of $\delta \lambda$ is obtained only at $T \gg$ $E_{\text {nonlin }}, E_{\text {nonloc }}$, and will be renormalized to $T^{2}$ whenever $E_{\text {nonloc }}$ or $E_{\text {nonlin }}$ is larger than $T$. The linear $H$ dependence of $\delta \lambda$ predicted by Yip and Sauls, the socalled nonlinear Meissner effect, is recovered for $E_{\text {nonlin }} \gg T, E_{\text {nonloc }}$, but

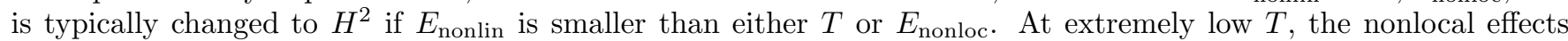
cutoff the nonlinear effect at $E_{\text {nonlin }}<E_{\text {nonloc }}$. This happens when $H<H^{*}$. Both $E_{\text {nonlin }}$ and $E_{\text {nonloc }}$ turn out to be geometry dependent. When the magnetic field is along the $\hat{c}$ axis and the angle $\theta$ that the supercurrent makes with the $\hat{a}$ axis is not near a nodal value, $H^{*} \simeq H_{c 1}$, leading to the unobservability of the nonlinear Meissner effect. When $\theta$ is near a nodal value, $E_{n o n l o c}$ is so small that the nonlinear Meissner effect may be recovered. However the effects on $\lambda(H, T)$ of Andreev bound states, neglected in this work, need to be better understood before this conclusion can be taken seriously.

Are any of the above predictions supported by experiment? While the linear- $T$ temperature dependence in the penetration depth measurements above $1 \mathrm{~K}$ in clean samples of cuprate superconductors has been observed for several years, nonlinear and nonlocal consequences of the simple $d$-wave theory of electromagnetic response in the Meissner state have proven more difficult to confirm. In zero external field, any low- $T \delta \lambda \sim T^{2}$ behavior observed until now occurs in an experimental situation where the result can more plausibly be attributed to impurities. Recent highresolution resonant coil measurements have indeed identified linear field dependences $\delta \lambda \simeq b H$ with coefficients $b$ at temperatures $T \simeq 1 \mathrm{~K}$ not too different from the predictions of the local, nonlinear theory. However, the temperature dependence in such cases is opposite to that predicted, and the linear field dependence is not always observed. This suggests that earlier reports of the observation of the nonlinear Meissner effect displaying larger linear- $H$ terms in lower resolution experiments must be treated with skepticism. Other manifestations of the nonlinear Meissner effect, e.g., the ac transverse magnetization measurements of Bhattacharya et al. [21] have also failed to observe the predicted angular harmonics, [14.33 and it is tempting to conclude that a single mechanism is responsible for the null result obtained by all types of experiments.

What is the nature of this mechanism? Our proposal that the linear field dependence must generically be supressed at fields $H \simeq H_{c 1}$ clearly does not provide a complete explanation for the failure to observe the nonlinear Meissner effect, as no $H^{2}$ dependence has been reported in any sample. There appear to us to be two classes of explanation for the existing data on $\delta \lambda(H)$ and transverse magnetization. First, as suggested by Carrington et al., [19] a few vortices trapped in the surface layer can give rise to a "spurious" contribution to $\delta \lambda(H)$ due to the microwave excitations of these vortices in their pinning potentials (Campbell pinning penetration depth). [37] Depending on the size of the pinning force, this can give a result, $\propto H$, of roughly the correct magnitude. It is not clear whether the transverse magnetization experiments can be quantitatively explained by such a contribution. 
The second possible explanation for the disagreement of the experiments with both the local and nonlocal $d$ wave theories was put forward by Bhattacharya et al., [21] who suggested the existence of a small bulk subdominant order parameter component, e.g., of $s$ symmetry, which condenses in a state $\pi / 2$ out of phase with the dominant $d$ component. Such a state has a true "minigap" of order the size of the $s$ component. The authors of Ref. 21] estimated that a minigap of a few degrees $K$ would be sufficient to eliminate the nonlinear signal in their experiments on YBCO. In such a scenario, any experiment which depends on the existence of quasiparticles well below the minigap scale must report a null result. Several other experiments have reported significant quasiparticle densities well below this temperature. In particular, the thermal conductivity measurements of Taillefer et al. [36] reveal the existence of a normal-fluid density in both the YBCO and BSCCO systems down to at least $50 \mathrm{mK}$. We therefore do not believe this proposal is tenable.

An argument against the importance of nonlocal effects in recent experiments with $H \| a b$ which may provide a clue to the origins of the spurious signal has been given recently by Bhattacharya et al. [32] These authors point out that the field of first flux entry, $H_{c 1}^{*} \simeq 250-300$ Oe, appears to be an order of magnitude larger than the Ginzburg-Landau lower critical field $H_{c 1} \simeq 30-50$ Oe, of order the crossover field between nonlocal and local field dependence for YBCO in this geometry. There should therefore, according to these authors, be a rather large intermediate field region before flux penetrates the sample where the local theory applies and the nonlinear Meissner effect should be observable. This argument, while intriguing, begs the question of why the observed field of first flux penetration is so different from the Ginzburg-Landau $H_{c 1}$ [34]. The obvious explanation is a geometrical surface barrier effect of the type discussed by Bean and Livingston, and by De Gennes. 35] Vortices which nucleate in the surface layer in the intermediate field range, while thermodynamically stable in the bulk, are expelled by an image potential due to the surface. The intermediate field "Meissner" state is therefore thermodynamically unstable, and vortices may be easily trapped at the sample corners or within the skin depth. Two recent high-resolution penetration depth measurements [19,20] failed to measure the predicted intrinsic (nonlinear) temperature dependence in this field range, but did observe a large linear- $H$ field dependence most likely attributable to trapped vortices. On the other hand, the lack of hysteresis observed by Bhattacharya et al. when cycling above $H_{c 1}^{*}$ argues against a significant number of pinned vortices. Nevertheless, the thermodynamic instability of the Meissner state suggests that the existence of a region of perfectly laminar surface flow with $H_{c 1}<H<H_{c 1}^{*}$ where the simple theories presented above apply may be too naive. The correct picture of the surface layer in such a situation may be one of fluctuating supercurrents whose time averaged effect on those quasiparticles present may be similar to that of trapped vortices. These fluctuations would make the field penetration layer instantaneously highly nonuniform, potentially leading to a broadening and a redistribution of spectral weight of the predicted transverse magnetization harmonics. 14] We believe the only field regime where one can safely assume a true Meissner response is for fields below the thermodynamic $H_{c 1}$, i.e., in the nonlocal response regime.

We propose two types of experiments which could help clarify the present impasse regarding theoretical interpretation of experimental results. The first is a direct measurement of the magnetization using a dc superconducting quantum interference device. This has not been yet performed as a function of applied magnetic field, to our knowledge. If the requisite sensitivity can be obtained, such an experiment would have the advantage of eliminating the Campbell penetration depth contributions, which may be dominating the signal in the resonant coil experiments. 19,20 The second experiment, to maximize the possiblity of seeing nonlocal effects and eliminate the uncertainties in the analysis with $H \| a b$, would be a measurement with field $H \| c$ on a long cylindrical sample with geometrical axis coinciding with the crystalline $\hat{c}$ axis. These may help to settle this long-standing controversy.

\section{ACKNOWLEDGMENTS}

The authors are grateful to A. Carrington, F. Evers, M. Fogelström, M. Franz, R. Giannetta, A. Goldman, S. Kirchner, J. Kroha, N. Schopohl, and Y.-J. Wang for helpful communications. Partial support was provided by the A. v. Humboldt Foundation, NSF Grant No. DMR-9974396, and the Deutsche Forschungsgemeinschaft.

\section{APPENDIX: RENORMALIZATION OF THE PENETRATION DEPTH}

In Sec. IV, we calculated the penetration depth in the presence of a constant $v_{s}$. In a real system, however, the $v_{s}$ field of the screening current is varying in space. The correction to the penetration depth due to the spatially varying nature of $v_{s}$ needs to be examined in order to check the validity of the qualitative conclusions we have reached. Note that the rigorous result can only be obtained through the solution of the full self-consistent magnetostatic problem, 
from which analytical information is difficult to obtain. However, we can improve our previous results by employing better approximations. We may imagine the system to be subdivided into many layers, in each of which $v_{s}$ is roughly constant. But $v_{s}$ in different layers is not the same. The response function in real space is now approximatedly defined as

$$
\mathcal{K}\left(y, y^{\prime}\right) \simeq \mathcal{K}\left[y-y^{\prime}, v_{s}\left(\frac{y+y^{\prime}}{2}\right)\right] .
$$

The only terms not included in this approximation involve gradients of $v_{s}$. However, in the regime where $E_{n o n l i n} \gg$ $E_{\text {nonloc }}$, we estimate $\nabla \mathbf{v}_{s} / m \ll v_{s}^{2}$ and hence gradients of $v_{s}$ are negligible where the nonlinear effect is important at all.

According to $\mathbf{A}=(m c / e) \mathbf{v}_{s}$, Eq. (61) can be rewritten in an operator representation

$$
v_{s}=-\frac{4 \pi e}{m c^{2}}\left(\nabla^{2}-\frac{4 \pi}{c} \hat{\mathcal{K}}\right)^{-1} j_{\text {ext }} .
$$

Using the expansion

$$
\frac{4 \pi}{c} \hat{\mathcal{K}} \simeq \lambda_{0}^{-2}+\frac{4 \pi}{c} \delta \hat{\mathcal{K}}
$$

we get

$$
v_{s} \simeq-\frac{4 \pi e}{m c^{2}}\left[\left(\nabla^{2}-\lambda_{0}^{-2}\right)^{-1} j_{e x t}+\left(\nabla^{2}-\lambda_{0}^{-2}\right)^{-1}\left(\frac{4 \pi}{c} \delta \hat{\mathcal{K}}\right)\left(\nabla^{2}-\lambda_{0}^{-2}\right)^{-1} j_{e x t}\right] .
$$

In the coordinate representation $v_{s}$ takes the form of

$$
\begin{gathered}
v_{s}(y) \simeq v_{s}^{(0)}(y)+v_{s}^{(1)}(y), \\
v_{s}^{(0)}(y)=-\frac{e H}{m c} \lambda_{0} e^{-|y| / \lambda_{0}} \\
v_{s}^{(1)}(y)=-\frac{8 e H}{m c^{2}} \int_{-\infty}^{\infty} \frac{d q_{1}}{2 \pi} \frac{e^{i q_{1} y}}{q_{1}^{2}+\lambda_{0}^{-2}} \int_{-\infty}^{\infty} d R \int_{-\infty}^{\infty} d q_{2} \frac{e^{i 2 R\left(q_{2}-q_{1}\right)}}{\left(2 q_{2}-q_{1}\right)^{2}+\lambda_{0}^{-2}} \delta \mathcal{K}\left(\mathbf{q}_{2}, \mathbf{v}_{s}(R)\right) .
\end{gathered}
$$

At this stage, the $\mathbf{q}$ and $\mathbf{v}_{s}$ dependence of $\delta \mathcal{K}$ can be approximately taken to be the same as what we got in Sec. II, with the point kept in mind that $v_{s}$ is a function of space which needs to be solved self-consistently based on Eqs. (78)-(80). Using the definition in Eq. (59) and the relation $H(y)=(m c / e)\left(d v_{s}(y) / d y\right)$, we find

$$
\lambda_{\text {spec }}=-\frac{m c}{e H} v_{s}(0)
$$

which turns out to be

$$
\lambda_{\text {spec }} \simeq \lambda_{0}-\frac{8}{c} \int_{-\infty}^{\infty} \frac{d q_{1}}{2 \pi} \frac{1}{q_{1}^{2}+\lambda_{0}^{-2}} \int_{-\infty}^{\infty} d R \int_{-\infty}^{\infty} d q_{2} \frac{e^{i 2 R\left(q_{2}-q_{1}\right)}}{\left(2 q_{2}-q_{1}\right)^{2}+\lambda_{0}^{-2}} \delta \mathcal{K}\left(\mathbf{q}_{2}, \mathbf{v}_{s}(R)\right) .
$$

It is easy to check that in the case of a constant $v_{s}$, the penetration depth in Eq. (82) reduces exactly to that in Eq. (62).

Now we insert Eqs. (33) and (48) into Eq. (82) to obtain the normalized change in the penetration depth

$$
\begin{gathered}
\frac{\delta \lambda_{\text {spec }}}{\lambda_{0}}=\frac{T}{\pi \lambda_{0} \Delta_{0}} \sum_{l= \pm 1} u_{\theta l}^{2} \int_{-\infty}^{\infty} \frac{d t_{1}}{2 \pi} \int_{-\infty}^{\infty} d R \frac{I_{\theta l}\left(t_{1}, R\right)}{t_{1}^{2}+1}, \\
I_{\theta l}\left(t_{1}, R\right)=\int_{0}^{2\left|h_{\theta l}(R)\right|} d t_{2}\left[\frac{e^{i 2\left(t_{2}-t_{1}\right) R / \lambda_{0}}}{\left(2 t_{2}-t_{1}\right)^{2}+1}+\frac{e^{i 2\left(t_{2}+t_{1}\right) R / \lambda_{0}}}{\left(2 t_{2}+t_{1}\right)^{2}+1}\right] \beta_{\theta l}\left|v_{s}(R)\right| \\
+\int_{2\left|h_{\theta l}(R)\right|}^{\infty} d t_{2}\left[\frac{e^{i 2\left(t_{2}-t_{1}\right) R / \lambda_{0}}}{\left(2 t_{2}-t_{1}\right)^{2}+1}+\frac{e^{i 2\left(t_{2}+t_{1}\right) R / \lambda_{0}}}{\left(2 t_{2}+t_{1}\right)^{2}+1}\right]\left[\frac{\pi}{4} \frac{a_{\theta l} t_{2}}{\lambda_{0}}+\beta_{\theta l}\left|v_{s}(R)\right| \lambda_{0}^{2} \frac{\beta_{\theta l}^{2} v_{s}^{2}(R)+\pi^{2}}{6 a_{\theta l}^{2} t_{2}^{2}}\right],
\end{gathered}
$$


where $a_{\theta l}=v_{F} u_{\theta,-l} /(2 \sqrt{2} T)$ and $\beta_{\theta l}=m v_{F} u_{\theta l} /(\sqrt{2} T)$. Clearly the nonlinear and the nonlocal corrections to the penetration depth are involved in $I_{\theta l}\left(t_{1}, R\right)$.

For $h \gg 1$ it is straightforward to find that the leading correction to the penetration depth is linear in $H$. It is given by the first integral in $I_{\theta l}\left(t_{1}, R\right)$ with $v_{s}(R)$ replaced by $v_{s}^{(0)}(R)$ and the upper bound of the integral $2\left|h_{\theta l}(R)\right|$ expanded to $\infty$. After proceeding with this one gets

$$
\frac{\delta \lambda_{\text {spec }}}{\lambda_{0}} \simeq \frac{\zeta_{\theta}}{\sqrt{2}} \frac{H}{H_{0}}=\frac{\pi \zeta_{\theta}}{3 \sqrt{2}} \kappa^{-1} h, \quad h_{\theta l} \gg 1, \quad l= \pm 1 .
$$

Comparing with the first term in Eq. (72) we find that an extra prefactor $2 / 3$ is acquired through this renormalization scheme. When we compare this result with Yip-Sauls result (四), however, as we mentioned at the end of Sec. IV A, we note a different definition for the penetration depth is used in their paper, and an extra prefactor 2 should be expected. This is to say that if we use the definition of Yip-Sauls for the penetration depth, we should find it 2 times as large as that in Eq. (85). The reason that the expected 2 is actually missing here is again the consequence of the perturbation treatment of the coupling of the quasiparticles to the $\mathbf{A}_{\mathbf{q} \neq 0}$ mode, the detailed discussion of which has been presented in Sec. III.

For $h \ll 1$, the leading correction to the penetration depth is the contribution from the nonlocal effects and is independent of $H$. It can be obtained from the second integral in $I_{\theta l}\left(t_{1}, R\right)$ by setting the lower bound $2 h_{\theta l}(R)$ to be 0 . The leading $H$-dependent correction to the penetration depth is found to be quadratic of $H$. After some algebra we get

$$
\frac{\delta \lambda_{\text {spec }}}{\lambda_{0}} \simeq \frac{\pi}{8 \sqrt{2}} \sum_{l= \pm 1} d_{\theta l 1} \kappa^{-1}+c_{1} \lambda_{0} h^{2} \kappa^{-1} \sum_{l= \pm 1} \frac{u_{\theta l}^{4}}{u_{\theta,-l}}, \quad h_{\theta l} \ll 1, \quad l= \pm 1 .
$$

We find that the first term, i.e., the correction from the nonlocal effects is exactly the same as the first term in Eq. (71). This means, as expected, that the nonlocal effects are not influenced by the spatial varying nature of $v_{s}$. The second term coincides qualitatively with that in Eq. (71), but acquires an insignificant extra prefactor through the renormalization scheme. If this expansion were continued, it is clear the result obtained would converge to the result of Yip and Sauls [12] in the local limit.

[1] D. Vollhardt and P. Wölfle, The Superfluid Phases of Helium 3 (Taylor \& Francis, London, 1990).

[2] F. Gros, B. S. Chandrasekhar, D. Einzel, P. J. Hirschfeld, and K. Andres, Z. Phys. B 64, 175 (1986).

[3] W. N. Hardy, D. A. Bonn, D. C. Morgan, Ruixing Liang, and Kuan Zhang, Phys. Rev. Lett. 70,3999 (1993).

[4] I. Kosztin and A. J. Leggett, Phys. Rev. Lett. 79, 135 (1997).

[5] W. O. Putikka, P. J. Hirschfeld, and P. Wölfle, Phys. Rev. B 41, 7285 (1990).

[6] The effects of nonlocal electrodynamics on the real part of the microwave conductivity, or surface resistance, have also been investigated. In general these effects have also been found to be small, except at very low energies. See, e.g., C. Zuccaro, C. T. Rieck, and K. Scharnberg, Physica C 235-240, 1807 (1994); S. Hensen, G. Müller, C. T. Rieck, and K. Scharnberg, Phys. Rev. B 56, 6237 (1997).

[7] A. Hosseini, R. Harris, Saeid Kamal, P. Dosanjh, J. Preston, Ruixing Liang, W. N. Hardy, and D. A. Bonn, Phys. Rev. B 60, 1349 (1999).

[8] M. Prohammer and J. P. Carbotte, Phys. Rev. B 43, 5370 (1991).

[9] P.J. Hirschfeld and N. Goldenfeld, Phys. Rev. B 48, 4219 (1993).

[10] N. Schopohl and O. V. Dolgov, Phys. Rev. Lett. 80, 4761(1998).

[11] G. E. Volovik, Phys. Rev. Lett. 81, 4023 (1998).

[12] S. K. Yip and J. A. Sauls, Phys. Rev. Lett. 69, 2264 (1992).

[13] D. Xu, S. K. Yip, and J. A. Sauls, Phys. Rev. B 51, 16233 (1995).

[14] I. Zutić and T. Valls, Phys. Rev. B 58, 8738 (1998).

[15] M.H. S. Amin, I. Affleck, and M. Franz, Phys. Rev. B 58, 5848 (1998).

[16] J. Ferrer, M. A. González-Alvarez, and J. Sánchez-Cañizares, Superlattices and Microstructures 25, 1125 (1999).

[17] P. J. Hirschfeld, M.-R. Li, and P. Wölfle, Phys. Rev. Lett. 81, 4024 (1998).

[18] A. Maeda, Y. Iino, T. Hanaguri, N. Motohira, K. Kishio, and T. Fukase, Phys. Rev. Lett. 74, 1202 (1995); A. Maeda et al., J. Phys. Soc. Jpn. 65, 3638 (1996).

[19] A. Carrington, R. W. Giannetta, J. T. Kim, and J. Giapintzakis, Phys. Rev. B 59, R14173 (1999). 
[20] C. P. Bidinosti, W. N. Hardy, D. A. Bonn and Ruixing Liang, Phys. Rev. Lett. 83, 3277 (1999).

[21] A. Bhattacharya, I. Žutic, O. T. Valls, A. M. Goldman, U. Welp, and B. Veal, Phys. Rev. Lett. 82 , 3132 (1999).

[22] M.-R. Li, P. J. Hirschfeld, and P. Wölfle, Phys. Rev. Lett. 81, 5640 (1998).

[23] Usually there is an additional term representing onsite repulsion term that prevents an usual s-wave pairing. This term is important in studying the stability problem of a $d$-wave superconductor. But in this work we assume there is always $d$-wave pairing thus decoupling this term by a trivial number operator that can be simply absorbed into the chemical potential. Besides, throughout this paper, we choose $k_{B}=\hbar=1$ units.

[24] K. Maki and T. Tsuneto, Prog. Theor. Phys. 27, 228 (1962).

[25] A. A. Abrikosov, L. P. Gor'kov, and I. E. Dzyaloshinski, Methods of Quantum Field Theory in Statistical Physics (PrenticeHall, Englewood Cliffs, NJ, 1963).

[26] M. Tinkham, Introduction to Superconductivity (McGraw-Hill, New York, 1974).

[27] H. Walter et al., Phys. Rev. Lett. 80, 3598 (1998).

[28] M. Fogelström, D. Rainer and J. A. Sauls, Phys. Rev. Lett. 79, 281 (1997).

[29] D. I. Glazman and A. E. Koshelev, Zh. Eksp. Teor. Fiz. 97, 1371 (1990) [Sov. Phys. JETP 70, 774 (1990)].

[30] The expression for $H_{c 1}$ given here is a direct result of the anisotropic Ginzburg-Landau theory and is more accurate than that appearing in our paper (Ref. [22]).

[31] For representative values of $\xi_{0}$ and $\lambda_{0}$, see S. L. Cooper and K. E. Gray, in Physical Properties of High Temperature Superconductors, IV, edited by D. Ginsberg (World Scientific, Singapore, 1994).

[32] A. Bhattacharya, I. Žutić, O. T. Valls, and A. M. Goldman, Phys. Rev. Lett. 83, 887 (1999).

[33] I. Žutić and O. T. Valls, Phys. Rev. B 56, 1279 (1997).

[34] M.-R. Li, P. J. Hirschfeld, and P. Wölfle, Phys. Rev. Lett. 83, 888 (1999).

[35] C. P. Bean and J. D. Livingston, Phys. Rev. Lett. 12, 14 (1964); P. D. de Gennes, Solid State Commun. 3 , 127 (1965).

[36] L. Taillefer, B. Lussier, R. Gagnon, K. Behnia, and H. Aubin, Phys. Rev. Lett. 79, 483 (1997).

[37] M. W. Coffey and J. R. Clem, Phys. Rev. Lett. 67, 386 (1991); E. H. Brandt, ibid. 67, 2219 (1991).

[38] T. Dahm and D. J. Scalapino, Phys. Rev. B 60, 13125 (1999). 